\title{
Equilibria and Dislocations in Epitaxial Growth
}

\author{
I. Fonseca and G. Leoni \\ Department of Mathematical Sciences, \\ Carnegie Mellon University, \\ Pittsburgh, PA, U.S.A.
}

\author{
Massimiliano Morini \\ Università degli Studi di Parma \\ Parma, Italy
}

October 4, 2016

\begin{abstract}
An overview of recent analytical developments in epitaxial growth is presented. The energy release via the onset of dislocations is addressed. Regularity of quasistatic equilibria is studied both in the absence and in the presence of dislocations. Morphological evolution of anisotropic epitaxially strained films is considered under the assumption that the surface evolves by surface diffusion under the influence of a chemical potential.
\end{abstract}

\section{Contents}

1 Introduction 1

2 Existence Theory $\quad 4$

3 Regularity Theory 6

4 Second Variation and Minimality 10

$\begin{array}{llr}5 & \text { Dislocations } & 20\end{array}$

6 Evolution by surface diffusion with curvature regularization 23

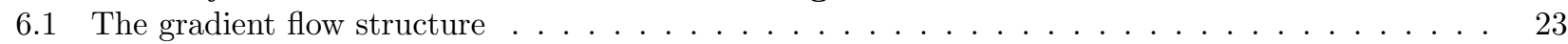

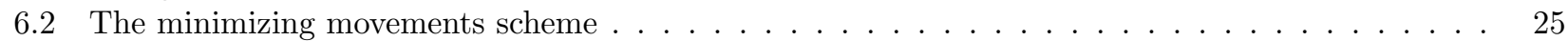

6.3 Local-in-time existence, uniqueness, and nonlinear stability of the flat configuration . . . . . . . 26

\section{Introduction}

In this paper we give an overview of recent analytical developments in the study of deposition of a crystalline film onto a substrate, in which the atoms of the film occupy natural lattice positions of the substrate. This process is called epitaxial growth. Here we are interested in eteropitaxy, i.e., epitaxy in the case when the film and the substrate have different crystalline structures.

At the onset of the deposition the atoms of the film tend to align themselves with those of the substrate because the energy gain associated with the chemical bonding effect is greater than the strain in the film due to the mismatch between the lattice parameters. As the film continues to grow, the stored strain energy per unit area of interface increases with the film thickness, rendering the flat layer of the film morphologically unstable or metastable, after a critical value of the thickness is reached. As a result, the free surface of the film becomes corrugated and the material agglomerates into clusters or isolated islands on the substrate. The formation of islands in systems such as In-GaAs/GaAs or SiGe/Si has important applications in high-end technology such as the fabrication of modern semiconductor electronic and optoelectronic devices (quantum dots laser). The Stranski-Krastanow (SK) growth mode occurs when the islands are separated by a thin wetting layer, while the Volmer-Weber (VW) growth mode refers to the case when the substrate is exposed between islands.

In what follows we adopt the two dimensional variational model considered by Spencer in [44] (see also [36], [45], and the references contained therein) and we follow the formulation given in [7]. We remark that 
two-dimensional configurations correspond to three-dimensional morphologies with planar symmetry. Although our presentation will be focused on the two-dimensional model, partial extensions of the results presented here to the three-dimensional setting can be found in [6] and [23].

We now describe the model more in detail. The free energy functional associated with the physical system is given by

$$
\int_{\Omega_{h}} Q(E(u)) d z+\int_{\Gamma_{h}} \psi(\nu) d \mathcal{H}^{1}
$$

where $z=(x, y) \in \mathbb{R}^{2}, h:(0, \ell) \rightarrow[0, \infty)$ is the function whose graph $\Gamma_{h}$ describes the profile of the film, assumed to be $\ell$-periodic, for some $\ell>0, \Omega_{h}$ is the region occupied by the film, i.e.,

$$
\Omega_{h}:=\{(x, y) \in(0, \ell) \times \mathbb{R}: 0<y<h(x)\},
$$

$u: \Omega_{h} \rightarrow \mathbb{R}^{2}$ is displacement of the material, $E(u):=\frac{1}{2}\left(\nabla u+\nabla^{T} u\right)$ is the linearized strain, the elastic energy density $Q: \mathbb{M}_{\mathrm{sym}}^{2 \times 2} \rightarrow[0,+\infty)$ is a positive definite quadratic form

$$
Q(\xi):=\frac{1}{2} \mathbb{C} \xi: \xi
$$

with $\mathbb{C}$ a positive definite fourth-order tensor so that $Q(\xi)>0$ for all $\xi \in \mathbb{M}_{\text {sym }}^{2 \times 2} \backslash\{0\}$, where : stands for the Euclidean inner product between $2 \times 2$ matrices, $\psi: \mathbb{R}^{2} \rightarrow[0, \infty)$ is an anisotropic surface energy density evaluated at the unit normal $\nu$ to $\Gamma_{h}$, and $\mathcal{H}^{1}$ denotes the one-dimensional Hausdorff measure. We suppose that $\psi$ is positively one-homogeneous and of class $C^{2}$ away from the origin, so that, in particular,

$$
\frac{1}{c}|\nu| \leq \psi(\nu) \leq c|\nu| \quad \text { for all } \nu \in \mathbb{R}^{2},
$$

for some $c>0$.

The substrate and the film admit different natural states corresponding to the mismatch between their respective crystalline structures. To be precise, a natural state for the substrate is attained at $u \equiv 0$, while a natural state for the film is given by $u \equiv A_{0} z$ for some nonzero $2 \times 2$ matrix $A_{0}$. Our models will reflect this mismatch either by setting the elastic bulk energy as $\int_{\Omega_{h}^{\infty}} W\left(E(u)-E_{0}\right) d z$, where $\Omega_{h}^{\infty}:=\{(x, y) \in(0, \ell) \times \mathbb{R}$ : $y<h(x)\}$,

$$
E_{0}(y):=\left\{\begin{array}{cl}
\frac{A_{0}+A_{0}^{T}}{2} & \text { if } y>0 \\
0 & \text { if } y \leq 0,
\end{array}\right.
$$

or by imposing in (1.1) the Dirichlet boundary condition of the form $u(x, 0) \equiv e_{0}(x, 0)$, for some constant $e_{0}$ measuring the mismatch between the lattices. In the exposition below, for the sake of presentation, we only consider the latter formulation.

The static theory to this problem was developed in [5], [7], [12], [20], [19], [27] in the two-dimensional case, and in [6], [9], [14] in three dimensions.

In Sections 2 and 3 we study existence and regularity of equilibrium solutions of the functional (1.1) in the linearly isotropic case and when the surface energy density is $\psi \equiv 1$. In particular, we show that if the period $\ell$ or the thickness of the film $d$ are sufficiently small, then the flat configuration $h=d / \ell$ is stable. Otherwise the film becomes corrugated and islands (quantum dots) are formed. In this case we can prove that the profile $h$ is smooth except for at most a finite number of vertical cuts and cusps, which are observed experimentally for thick films. In particular, the film meets the substrate at a zero contact angle.

In Section 4, we perform a finer analysis of some qualitative properties of the solutions and we establish some relevant local and global minimality results. The main tool will be the study of the second variation of the energy functional.

It has been observed in experiments that, for sufficiently thick films, a mechanism to release stress energy after the formation of cusps is the nucleation of dislocations(see, for instance, [17, 28, 32, 34, 47]). Moreover, dislocations migrate to the film/substrate interface, appear to arrange themselves in a periodic manner, and subsequently the film surface relaxes towards a planar-like morphology. In Section 5 we will address these issues, as well as existence and regularity of the film profile in the presence of dislocations, and we will give conditions under which the nucleation of dislocations is energetically favorable.

In the final part of the paper we study the morphological evolution of anisotropic epitaxially strained films and we assume that the surface evolves by surface diffusion under the influence of a chemical potential $\mu$. 
Further, mass transport in the bulk occurs at a much faster time scale (see [38]) and thus will be neglected in our evolution equations. Concerning the surface diffusion mechanism, we recall that according to the Einstein-Nernst relation, the surface flux of atoms is proportional to the tangential gradient $\nabla_{\sigma} \mu$ of the chemical potential. In turn, the divergence of the surface flux equals the rate at which material is removed from or deposited on the interface, due to mass conservation. Thus, we are led to the following evolution volume preserving law:

$$
V=C \mu_{\sigma \sigma},
$$

where $C>0$ is a material constant, $V$ denotes the normal velocity of the evolving profile, and $(\cdot)_{\sigma}$ stands for the tangential derivative along the curve. Moreover, the chemical potential $\mu$ can be expressed as the first variation (with respect to the profile variable) of the free-energy functional (1.1). In our context, this becomes (assuming $C=1)$

$$
V=\left(k_{\psi}+Q(E(u))\right)_{\sigma \sigma},
$$

where $k_{\psi}$ is the anisotropic curvature of $\Gamma_{h}$ (see $(4.34)$ ) and $u(\cdot, t)$ is the elastic equilibrium in $\Omega_{h(\cdot, t)}$, i.e., the minimizer of the elastic energy under the prescribed periodicity and boundary conditions (see (1.6) below).

If the surface energy density $\psi$ is highly anisotropic, there may be directions $\nu$ for which

$$
D^{2} \psi(\nu)[\tau, \tau]>0 \quad \text { for all } \tau \perp \nu, \tau \neq 0
$$

fails, see for instance [16], [43]. In this case, the evolution equation (1.4) is backward parabolic. In order to overcome this ill-posedness, a common approach found in the literature is to regularize with higher order terms, i.e, to consider in (1.1) a curvature-dependent surface energy $\hat{\psi}(\nu, k)$, where $k$ is the standard curvature. This was first suggested by Herring ([33]) based on physical considerations. This approach was later adopted in [4] and [16], by considering surface energies of the form

$$
\hat{\psi}(\nu, k)=\psi(\nu)+\frac{\varepsilon}{2} k^{2},
$$

with $\varepsilon$ a (small) positive constant. Note that the additional term $\frac{\varepsilon}{2} k^{2}$ may be thought of as a curvature regularization of the non-convex surface density $\psi$. This leads to the following singular perturbation of the surface energy

$$
\int_{\Gamma_{h}}\left(\psi(\nu)+\frac{\varepsilon}{2} \kappa^{2}\right) d \mathcal{H}^{1}
$$

Taking into account the additional terms coming from the first variation of the curvature regularization, (1.4) should be now replaced by the volume preserving evolution law

$$
V=\left(k_{\psi}+Q(E(u))-\varepsilon\left(k_{\sigma \sigma}+\frac{1}{2} k^{3}\right)\right)_{\sigma \sigma} .
$$

Coupling this evolution equation on the profile of the film with the elastic equilibrium elliptic system holding in the film, and parametrizing $\Gamma$ using $h: \mathbb{R}^{2} \times\left[0, T_{0}\right] \rightarrow(0, \infty)$, we obtain the following Cauchy system of equations with initial and natural boundary conditions:

$$
\left\{\begin{array}{l}
\frac{1}{J} \frac{\partial h}{\partial t}=\left(k_{\psi}+Q(E(u))-\varepsilon\left(k_{\sigma \sigma}+\frac{1}{2} k^{3}\right)\right)_{\sigma \sigma}, \quad \text { in } \mathbb{R} \times\left[0, T_{0}\right], \\
\operatorname{div} \mathbb{C} E(u)=0 \quad \text { in } \Omega_{h}, \\
\mathbb{C} E(u)[\nu]=0 \text { on } \Gamma_{h}, \quad u(x, 0, t)=e_{0}(x, 0), \\
h(\cdot, t) \text { and } \nabla u(\cdot, t) \quad \text { are } \ell \text {-periodic, } \\
h(\cdot, 0)=h_{0},
\end{array}\right.
$$

where we set

$$
J:=\sqrt{1+\left|\frac{\partial h}{\partial x}\right|^{2}} .
$$

and $h_{0} \in H_{\text {loc }}^{2}(\mathbb{R})$ is a $\ell$-periodic function.

We will address the gradient flow structure of the geometric flow, the local-in-time existence and uniqueness of the solution to the corresponding initial value problem in the context of evolving films (one-dimensional graphs), and the long-time behavior of the solution for a suitable class of initial data. We will use the gradient 
flow structure of (1.6) with respect to a suitable $H^{-1}$-metric (see, e.g., [11]) to solve the equation via a minimizing movement scheme (see [2]), i.e., we will discretize the problem in time and solve suitable minimum incremental problems.

If instead of $H^{-1}$ we used the gradient flow with respect to an $L^{2}$-metric, we would obtain a fourth order evolution equation describing motion by evaporation-condensation (see [11], [31], and [40]).

Sixth-order evolution equations of this type may be found in literature in related contexts (see, e.g., [31] without elasticity, see [43] for the evolution of voids in elastically stressed materials, and [42], [10]). However, short time existence of solutions to (1.6) obtained in [21], [23] are the first such results for geometric surface diffusion equations with elasticity in two and three dimensions. The results summarized in this paper can be found in [20], [21], [23], [24].

\section{Existence Theory}

We start by introducing the class of admissible film profiles over the interval $(0, \ell)$, which are functions with finite total variation in $(0, \ell)$ whose $\ell$-periodic extensions are lower semicontinuous (l.s.c.) in $\mathbb{R}$. Throughout the paper we will identify these functions with their periodic extensions. Thus, we set

$$
A P(0, \ell):=\{h: \mathbb{R} \rightarrow[0,+\infty): h \text { is l.s.c. and } \ell \text {-periodic, } \operatorname{Var}(h ; 0, \ell)<+\infty\},
$$

where $\operatorname{Var}(h ; 0, \ell)$ denotes the pointwise total variation of $h$ over the interval $(0, \ell)$. We will also use the notation

$$
h^{+}(x):=\max \{h(x+), h(x-)\}, \quad h^{-}(x):=\min \{h(x+), h(x-)\}
$$

where $h(x+)$ and $h(x-)$ denote the right and left limits, respectively, at $x$.

The reference configuration $\Omega_{h}$ of the film is represented by the subgraph of $h$ within the stripe $(0, \ell) \times \mathbb{R}$. To be precise,

$$
\Omega_{h}:=\{(x, y): x \in(0, \ell), 0<y<g(x)\}, \quad \Omega_{h}^{\#}:=\{(x, y): x \in \mathbb{R}, 0<y<h(x)\}
$$

while the profile of the film is given by

$$
\Gamma_{h}:=\left\{(x, y): x \in[0, \ell), h^{-}(x) \leq y \leq h^{+}(x)\right\} .
$$

Moreover, we introduce the set

$$
\Sigma_{h}:=\left\{(x, y): x \in[0, \ell), h(x)<h^{-}(x), h(x) \leq y \leq h^{-}(x)\right\}
$$

Note that $\Sigma_{h}$ is made up of at most countably many vertical segments and represents the collection of all vertical cracks that have possibly formed within the film material.

We will also use the notation

$$
\Gamma_{h}^{\#}:=\left\{(x, y) \in \mathbb{R}^{2}: x \in \mathbb{R}, h^{-}(x) \leq y \leq h^{+}(x)\right\}
$$

and, similarly, one can define the set $\Sigma_{h}^{\#}$. Notice that $\Omega_{h}^{\#}, \Gamma_{h}^{\#}$, and $\Sigma_{h}^{\#}$ are simply the $\ell$-periodic extensions of $\Omega_{h}, \Gamma_{h}$, and $\Sigma_{h}$, respectively.

We now introduce a suitable convergence in $A P(0, \ell)$. To this aim, we recall that if $C_{1}, C_{2}$ are closed subsets of $\mathbb{R}^{2}$ their Hausdorff distance is defined as

$$
d_{H}\left(C_{1}, C_{2}\right):=\inf \left\{\varepsilon>0: C_{2} \subset \mathcal{N}_{\varepsilon}\left(C_{1}\right) \text { and } C_{1} \subset \mathcal{N}_{\varepsilon}\left(C_{2}\right)\right\}
$$

where $\mathcal{N}_{\varepsilon}(C)$ denotes the $\varepsilon$-neighborhood of $C$.

Definition 2.1 (Convergence in $A P(0, \ell)$ ) We say that $h_{n} \rightarrow h$ in $A P(0, \ell)$ if

$$
\sup _{n} \operatorname{Var}\left(h_{n} ; 0, \ell\right)<+\infty \quad \text { and } \quad d_{H}\left(\mathbb{R}_{+}^{2} \backslash \Omega_{h_{n}}^{\#}, \mathbb{R}_{+}^{2} \backslash \Omega_{h}^{\#}\right) \rightarrow 0,
$$

where $\mathbb{R}_{+}^{2}=\left\{(x, y) \in \mathbb{R}^{2}: y \geq 0\right\}$. 
Remark 2.2 (Compactness) It can be shown that if $\left\{h_{n}\right\} \subset A P(0, \ell)$ and $\sup _{n} \operatorname{Var}\left(h_{n} ; 0, \ell\right)<+\infty$, then there exists $h \in A P(0, \ell)$ such that, up to a (not relabeled) subsequence, $h_{n} \rightarrow h$ in $A P(0, \ell)$ (in the sense of the previous definition). Moreover,

$$
h(x)=\inf _{x_{n} \rightarrow x} \liminf _{n \rightarrow \infty} h_{n}\left(x_{n}\right) .
$$

Next we introduce the class of admissible elastic displacements.

Given $h \in A P(0, \ell)$, we consider

$$
\begin{aligned}
& L D_{\#}\left(\Omega_{h} ; \mathbb{R}^{2}\right):=\left\{u \in L_{\mathrm{loc}}^{2}\left(\Omega_{h}^{\#} ; \mathbb{R}^{2}\right): u(x, y)=u(x+b, y) \text { for }(x, y) \in \Omega_{h}^{\#},\right. \\
& \left.\left.E(u)\right|_{\Omega_{h}} \in L^{2}\left(\Omega_{h} ; \mathbb{R}^{2}\right)\right\} .
\end{aligned}
$$

Finally, for a given mismatch strain $e_{0} \geq 0$, the space of admissible configurations is defined by

$$
\begin{aligned}
& X\left(e_{0} ; \ell\right):=\{(h, u): h \in A P(0, \ell), u: \Omega_{h}^{\#} \rightarrow \mathbb{R}^{2} \text { such that } \\
&\left.u(\cdot, \cdot)-e_{0}(\cdot, 0) \in L D_{\#}\left(\Omega_{h} ; \mathbb{R}^{2}\right), u(x, 0)=\left(e_{0} x, 0\right) \text { for all } x \in \mathbb{R}\right\} .
\end{aligned}
$$

It is useful to consider the following convergence in $X\left(e_{0} ; \ell\right)$.

Definition 2.3 We say that $\left(h_{n}, u_{n}\right) \rightarrow(h, u)$ in $X\left(e_{0} ; \ell\right)$ if $h_{n} \rightarrow h$ in $A P(0, \ell)$ in the sense of Definition 2.1 and $u_{n} \rightarrow u$ in $H_{\mathrm{loc}}^{1}\left(\Omega_{h}^{\#} ; \mathbb{R}^{2}\right)$.

Recall that $h_{n} \rightarrow h$ in $A P(0, \ell)$ implies $\mathbb{R}_{+}^{2} \backslash \Omega_{h_{n}}^{\#} \rightarrow \mathbb{R}_{+}^{2} \backslash \Omega_{h}^{\#}$ in the Hausdorff sense and thus for any $\Omega^{\prime} \subset \subset \Omega_{h}^{\#}$ we have $\Omega^{\prime} \subset \subset \Omega_{h_{n}}^{\#}$ for $n$ large enough. The last condition in Definition 2.3 is then equivalent to requiring that $u_{n} \rightarrow u$ in $H^{1}\left(\Omega^{\prime} ; \mathbb{R}^{2}\right)$ for any $\Omega^{\prime} \subset \subset \Omega_{h}^{\#}$, i.e., $u_{n} \rightarrow u$ in $H_{\text {loc }}^{1}\left(\Omega ; \mathbb{R}^{2}\right)$

Remark 2.4 Let $\left(h_{n}, u_{n}\right) \in X\left(e_{0} ; \ell\right)$ be such that

$$
\sup _{n}\left(\operatorname{Var}\left(h_{n} ; 0, \ell\right)+\left\|E\left(u_{n}\right)\right\|_{L^{2}\left(\Omega_{h_{n}}\right)}\right)<+\infty .
$$

Then there exists $(h, u) \in X\left(e_{0} ; \ell\right)$ such that, up to a (not relabeled) subsequence, $\left(h_{n}, u_{n}\right) \rightarrow(h, u)$ in $X\left(e_{0} ; \ell\right)$. To see this, apply Remark 2.2 to find $h \in A P(0, \ell)$ and a subsequence such that $h_{n} \rightarrow h$ in $A P(0, \ell)$. Let $\left\{A_{k}\right\}$ an increasing sequence of bounded Lipschitz domains such that $A_{k} \subset \subset \Omega_{h}^{\#}$ and $\partial A_{k} \cap\{y=0\}$ has positive measure for every $k \in \mathbb{N}$, and $A_{k} \nearrow \Omega_{h}^{\#}$. Since $u=\left(e_{0} x, 0\right)$ on $\partial A_{k} \cap\{y=0\}$ and $\left\{\left.E\left(u_{n}\right)\right|_{A_{k}}\right\}$ is bounded in $L^{2}$, a standard application of Korn's inequality yields that $u_{n}$ is bounded in $H^{1}\left(A_{k} ; \mathbb{R}^{2}\right)$ for every $k$. We may now apply a diagonalization argument to find $u$ such that $(h, u) \in X\left(e_{0} ; \ell\right)$ and (possibly extracting a further subsequence) $u_{n} \rightarrow u$ in $H_{\mathrm{loc}}^{1}\left(\Omega_{h}^{\#} ; \mathbb{R}^{2}\right)$.

For simplicity we will consider isotropic and homogeneous (linearly) elastic materials. Hence, the elastic energy density $Q: \mathbb{M}_{\text {sym }}^{2 \times 2} \rightarrow[0,+\infty)$ takes the form

$$
Q(\xi):=\frac{1}{2} \mathbb{C} \xi: \xi=\mu|\xi|^{2}+\frac{\lambda}{2}[\operatorname{tr}(\xi)]^{2},
$$

where

$$
\mathbb{C} \xi=\left(\begin{array}{cc}
(2 \mu+\lambda) \xi_{11}+\lambda \xi_{22} & 2 \mu \xi_{12} \\
2 \mu \xi_{12} & (2 \mu+\lambda) \xi_{22}+\lambda \xi_{11}
\end{array}\right) .
$$

Here $\mu$ and $\lambda$ are the so-called Lamé coefficients, which satisfy the ellipticity conditions

$$
\mu>0 \quad \text { and } \quad \lambda>-\mu .
$$

We finally associate to any admissible configuration $(h, u)$ a free-energy $F(h, u)$, given by the sum of the elastic energy stored in the film and the surface energy of the film profile. Following [7], we start by considering Lipschitz continuous profile functions. Precisely, given $(h, u) \in X\left(e_{0} ; \ell\right)$, with $h$ Lipschitz, we set

$$
F(h, u):=\int_{\Omega_{h}} Q(E(u)) d z+\mathcal{H}^{1}\left(\Gamma_{h}\right) .
$$




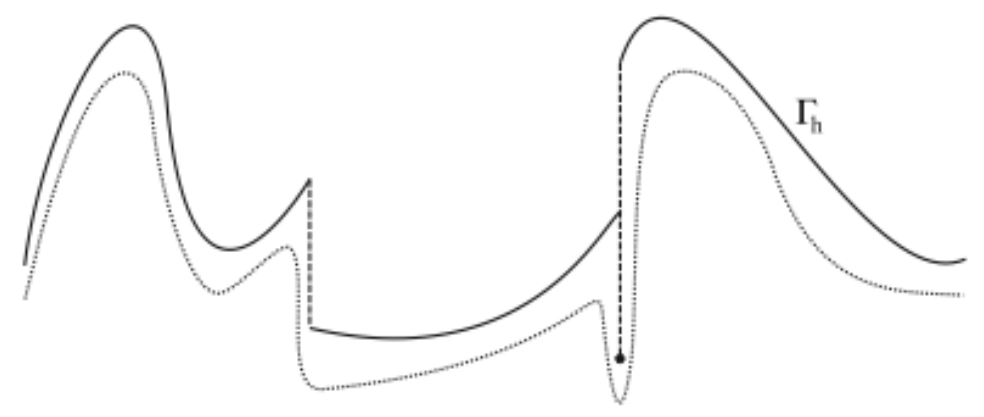

Figure 2.1: An irregular profile $h$ and a smooth approximation. The smooth profile surrounds the vertical crack so that in the limit its length is counted twice.

The extension of the definition of $F$ to general admissible pairs is obtained through a relaxation procedure: To this aim, for any $(h, u) \in X\left(e_{0} ; \ell\right)$ we set

$$
F(h, u):=\inf \left\{\liminf _{n} F\left(h_{n}, u_{n}\right):\left(h_{n}, u_{n}\right) \rightarrow(h, u) \text { in } X\left(e_{0} ; \ell\right), h_{n} \text { Lipschitz }\right\} .
$$

The following result, proved in [7] and in [20], provides a representation formula for $F$ in the general case and establishes the existence of a solution for the corresponding volume-constrained minimum problem.

Theorem 2.5 (Relaxation and existence) For any pair $(h, u) \in X\left(e_{0} ; \ell\right)$ we have

$$
F(h, u)=\int_{\Omega_{h}} Q(E(u)) d z+\mathcal{H}^{1}\left(\Gamma_{h}\right)+2 \mathcal{H}^{1}\left(\Sigma_{h}\right)
$$

Moreover, for any $d>0$ the minimum problem

$$
\min \left\{F(h, u):(h, u) \in X\left(e_{0} ;, \ell\right),\left|\Omega_{h}\right|=d\right\}
$$

has a solution.

We refer to [7] and [20] for the details of the proof of the representation formula. Here we just comment on the presence of the factor 2 multiplying the length of the vertical cracks $\Sigma_{h}$ : This is easily understood by observing that in the relaxation procedure any such crack is surrounded by a regular (Lipschitz) profile and thus, in the limit, its length must be counted twice (see Figure 2.1). Concerning the existence part, it follows from a standard application of the Direct Method of Calculus of Variations, using the compactness properties stated in Remark 2.4 and the lower semicontinuity of $F$ with respect to the convergence in $X\left(e_{0} ; \ell\right)$.

We conclude this subsection by introducing the following notation, that will be used throughout the paper: Given $h \in A P(0, \ell)$, we denote by $u_{h}$ the elastic equilibrium in $\Omega_{h}$ under the appropriate boundary conditions; i.e.,

$$
u_{h}:=\operatorname{argmin}\left\{\int_{\Omega_{h}} Q(E(v)) d z:(h, v) \in X\left(e_{0} ; \ell\right)\right\}
$$

\section{Regularity Theory}

In this section we review the regularity theory for (local) volume-constrained minimizers of (2.5), developed in [20] (see also [15]).

In order to state the main result, we need to introduce the set of cusp points of a function $h \in A P(0, \ell)$ :

$$
\Sigma_{h, c}:=\left\{(x, h(x)): x \in[0, \ell), h^{-}(x)=h(x), \text { and } h_{+}^{\prime}(x)=-h_{-}^{\prime}(x)=+\infty\right\},
$$

where $h^{-}$is defined in (2.1), while $h_{+}^{\prime}$ and $h_{-}^{\prime}$ stand for the right and left derivatives, respectively. As before, the set $\Sigma_{h, c}^{\#}$ is obtained by replacing $[0, \ell)$ by $\mathbb{R}$ in the previous formula and coincides with the $\ell$-periodic extension of $\Sigma_{h, c}$. 
Theorem 3.1 (Regularity of local minimizers) Let $(\bar{h}, \bar{u}) \in X\left(e_{0} ; \ell\right)$ be a minimizer for (2.5). Then the following regularity results hold:

(i) cusp points and vertical cracks are at most finite in $[0, \ell)$, i.e.,

$$
\text { card }\left(\left\{x \in[0, \ell):(x, y) \in \Sigma_{\bar{h}} \cup \Sigma_{\bar{h}, c} \text { for some } y \geq 0\right\}\right)<+\infty \text {; }
$$

(ii) the curve $\Gamma_{\bar{h}}^{\#}$ is of class $C^{1}$ away from $\Sigma_{\bar{h}}^{\#} \cup \Sigma_{\bar{h}, c}^{\#}$ and

$$
\lim _{x \rightarrow x_{0}^{ \pm}} \bar{h}^{\prime}(x)= \pm \infty \quad \text { for every } x_{0} \in \Sigma_{\bar{h}}^{\#} \cup \Sigma_{\bar{h}, c}^{\#}
$$

(iii) $\Gamma_{\bar{h}}^{\#} \cap\{\bar{h}>0\}$ is of class $C^{1, \alpha}$ away from $\Sigma_{\bar{h}}^{\#} \cup \Sigma_{\bar{h}, c}^{\#}$ for all $\alpha \in(0,1 / 2)$;

(iv) let $A:=\{x \in \mathbb{R}: \bar{h}(x)>0$ and $\bar{h}$ is continuous at $x\}$. Then $A$ is an open set of full measure in $\{\bar{h}>0\}$ and $\bar{h}$ is analytic in $A$.

Remark 3.2 Statement (ii) of Theorem 3.1 implies in particular that the zero contact angle condition between film and substrate holds.

The proof of Theorem 3.1 is quite long and technical and we will only outline the main steps and ideas below. Before doing so, we start with two preliminary lemmas. In the first one we show that the volume constraint can be replaced by a volume penalization, thus allowing more flexibility in the class of admissible variations.

Lemma 3.3 (Penalization) Let $(\bar{h}, \bar{u}) \in X\left(e_{0} ; \ell\right)$ be a minimizer for the problem (2.5). Then, for all $\Lambda>$ $Q_{0}:=(2 \mu+\lambda) e_{0}^{2}$ we have that $(\bar{h}, \bar{u})$ is a minimizer for the (unconstrained) problem

$$
\min \left\{F(h, u)+\Lambda|| \Omega_{h}|-d|:(h, u) \in X\left(e_{0} ; \ell\right)\right\} .
$$

Proof. We argue by contradiction. If $\left|\Omega_{\bar{h}}\right|<d$, we define $\tilde{h}:=\bar{g}+\left(d-\left|\Omega_{\bar{h}}\right|\right) / \ell$, and for all $(x, y) \in \Omega_{\tilde{h}}$

$$
\tilde{u}(x, y):= \begin{cases}\left(e_{0} x, 0\right) & \text { if } 0<y<\frac{d-\left|\Omega_{\bar{h}}\right|}{\ell}, \\ \bar{u}\left(x, y-\frac{d-\left|\Omega_{\bar{h}}\right|}{\ell}\right) & \text { if } y \geq \frac{d-\left|\Omega_{\bar{h}}\right|}{\ell} .\end{cases}
$$

Then, an explicit computation yields

$$
\begin{aligned}
F(\tilde{h}, \tilde{u})+\Lambda|| \Omega_{\tilde{h}}|-d|-F(\bar{h}, \bar{u})-\Lambda|| \Omega_{\bar{h}}|-d| & \\
& =(2 \mu+\lambda) e_{0}^{2}\left(d-\left|\Omega_{\bar{h}}\right|\right)-\Lambda\left(d-\left|\Omega_{\bar{h}}\right|\right)<0,
\end{aligned}
$$

which is a contradiction to the minimality of $(\bar{h}, \bar{u})$.

On the other hand, if $\left|\Omega_{\bar{h}}\right|>d$ then we may truncate $\bar{h}$ in such a way that the resulting function $\tilde{h}$ satisfies the constraint $\left|\Omega_{\tilde{h}}\right|=d$. In turn, we would obtain

$$
F(\tilde{h}, \bar{u})+\Lambda|| \Omega_{\tilde{h}}|-d|<F(\bar{h}, \bar{u})+\Lambda|| \Omega_{\bar{h}}|-d|,
$$

which again gives a contradiction to the minimality of $(\bar{h}, \bar{u})$. have

Next we show that the profile of a minimizer satisfies a uniform inner ball condition. To be precise, we

Lemma 3.4 (Uniform inner ball condition) Let $\Lambda>0$ and let $(\bar{h}, \bar{u}) \in X\left(e_{0} ; \ell\right)$ be a minimizing configuration for the problem (3.1). If $\varrho<1 / \Lambda$ then for all $z \in \Gamma_{\bar{h}}^{\#} \cup \Sigma_{\bar{h}}^{\#}$ there exists a ball $B_{\varrho}\left(z_{0}\right) \subset \Omega_{\bar{h}}^{\#} \cup(\mathbb{R} \times(-\infty, 0])$ such that $\partial B_{\varrho}\left(z_{0}\right) \cap\left(\Gamma_{\bar{h}}^{\#} \cup \Sigma_{\bar{h}}^{\#}\right)=\{z\}$. 
Proof. We split the proof into two steps.

Step 1. (Preliminary estimate) We start by showing the following fact:

Let $h \in A P(0, \ell)$ be nonnegative, let $B_{\varrho}\left(z_{0}\right)$ be a ball such that $B_{\varrho}\left(z_{0}\right) \subset\{(x, y): x \in \mathbb{R}$ and $y<h(x)\}$, and let $z_{1}=\left(x_{1}, y_{1}\right)$ and $z_{2}=\left(x_{2}, y_{2}\right)$ points in $\partial B_{\varrho}\left(z_{0}\right) \cap\left(\Gamma_{h}^{\#} \cup \Sigma_{h}^{\#}\right)$. Denote by $\gamma$ be the shortest arc on $\partial B_{\varrho}\left(z_{0}\right)$ connecting $z_{1}$ and $z_{2}$ (the upper one if $z_{1}$ and $z_{2}$ are antipodal) and let $\tilde{\gamma}$ be the arc on $\Gamma_{h}^{\#} \cup \Sigma_{h}^{\#}$ connecting $z_{1}$ and $z_{2}$. Finally, let $D$ be the region enclosed by $\gamma \cup \tilde{\gamma}$. Then

$$
\mathcal{H}^{1}(\tilde{\gamma})-\mathcal{H}^{1}(\gamma) \geq \frac{1}{\varrho}|D| .
$$

To see this, denote by $g$ the function on $\left[x_{1}, x_{2}\right]$ whose graph coincides with $\gamma$, and assume first that $h$ is Lipschitz continuous. Then, using the convexity of $t \mapsto \sqrt{1+t^{2}}$, the facts that $h\left(x_{1}\right)=g\left(x_{1}\right)$ and $h\left(x_{2}\right)=g\left(x_{2}\right)$ and that $-\left(\frac{g^{\prime}}{\sqrt{1+g^{\prime 2}}}\right)^{\prime}=\frac{1}{\varrho}$, we have

$$
\begin{aligned}
\mathcal{H}^{1}(\tilde{\gamma})-\mathcal{H}^{1}(\gamma) & =\int_{x_{1}}^{x_{2}}\left(\sqrt{1+h^{\prime 2}}-\sqrt{1+g^{\prime 2}}\right) d x \\
& \geq \int_{x_{1}}^{x_{2}} \frac{\left(h^{\prime}-g^{\prime}\right) g^{\prime}}{\sqrt{1+g^{\prime 2}}} d x \\
& =-\int_{x_{1}}^{x_{2}}(h-g)\left(\frac{g^{\prime}}{\sqrt{1+g^{\prime 2}}}\right)^{\prime} d x \\
& =\frac{1}{\varrho} \int_{x_{1}}^{x_{2}}(h-g) d x=\frac{1}{\varrho}|D| .
\end{aligned}
$$

The general case follows by approximating $h$ in $\left[x_{1}, x_{2}\right]$ with a sequence $h_{n}$ of Lipschitz functions and by passing to the limit in the above inequality.

Step 2. (Inner ball) We now show the inner ball condition for any radius $\varrho<1 / \Lambda$. It is not difficult to see that in order to establish such a condition it is enough to show that if $\varrho<1 / \Lambda$ and $B_{\varrho}\left(z_{0}\right) \subset\{(x, y): x \in$ $\mathbb{R}$ and $y<\bar{h}(x)\}$, then either $\partial B_{\varrho}\left(z_{0}\right) \cap\left(\Gamma_{\bar{h}}^{\#} \cup \Sigma_{\bar{h}}^{\#}\right)$ is a connected arc of circle contained in the upper half of $\partial B_{\varrho}\left(z_{0}\right)$ or $\partial B_{\varrho}\left(z_{0}\right) \cap\left(\Gamma_{\bar{h}}^{\#} \cup \Sigma_{\bar{h}}^{\#}\right)$ contains at most one point.

To this aim, we assume by contradiction that the first alternative does not hold and that $\partial B_{\varrho}\left(z_{0}\right) \cap\left(\Gamma_{\bar{h}}^{\#} \cup\right.$ $\left.\Sigma_{\bar{h}}^{\#}\right)$ contains at least two points $z_{1}=\left(x_{1}, y_{1}\right)$ and $z_{2}=\left(x_{2}, y_{2}\right)$, belonging to two different connected components of the set. Let $g$ and $\gamma$ be as in the previous step and denote by $\tilde{\gamma}$ the arc on $\Gamma_{\bar{h}}^{\#} \cup \Sigma_{\bar{h}}^{\#}$ connecting $z_{1}$ and $z_{2}$. We may modify $\bar{h}$ by replacing it with the function $\hat{h}$, which coincides with $\bar{h}$ in $[0, \ell) \backslash\left(x_{1}, x_{2}\right)$ and whose graph on $\left(x_{1}, x_{2}\right)$ is given by $\gamma$. Denote by $D$ the region enclosed by $\tilde{\gamma} \cup \gamma$ and note that $|D|>0$ by the assumption on $z_{1}$ and $z_{2}$. Then, using the estimate of the previous step, we have

$$
\begin{aligned}
F(\hat{h}, \bar{u})+\Lambda|| \Omega_{\hat{h}}|-d|- & F(\bar{h}, \bar{u})-\Lambda|| \Omega_{\bar{h}}|-d| \\
& \leq \mathcal{H}^{1}(\gamma)-\mathcal{H}^{1}(\tilde{\gamma})+\Lambda|D| \leq\left(\Lambda-\frac{1}{\varrho}\right)|D|<0,
\end{aligned}
$$

which gives a contradiction to the minimality of $(\bar{h}, \bar{u})$.

Remark 3.5 (Uniform ball condition of radius $1 / \Lambda$ ) From the previous lemma (letting $\leftrightharpoons 1 / \Lambda$ ) it readily follows that the following uniform ball condition of radius $1 / \Lambda$ holds: for all $z \in \Gamma_{\bar{h}}^{\#} \cup \Sigma_{\bar{h}}^{\#}$ there exists a ball $B_{1 / \Lambda}\left(z_{0}\right) \subset \Omega_{\bar{h}}^{\#} \cup(\mathbb{R} \times(-\infty, 0])$ such that $z \in \partial B_{\varrho}\left(z_{0}\right) \cap\left(\Gamma_{\bar{h}}^{\#} \cup \Sigma_{\bar{h}}^{\#}\right)$.

We are now ready to outline the strategy of the proof of Theorem 3.1. For the details we refer to [20] (see also [15]).

Outline of the proof of Theorem 3.1. We split the outline into several steps.

Step 1. (Penalization and inner ball condition) By Lemma 3.3 the pair $(\bar{h}, \bar{u})$ is an unconstrained minimizer of (3.1) for all $\Lambda>(2 \mu+\lambda) e_{0}^{2}$. We fix any such $\Lambda$ and we apply Lemma 3.4 (and Remark 3.5) to deduce that a uniform inner ball condition of radius $1 / \Lambda$ is satisfied at all points of $\Gamma_{\bar{h}}^{\#} \cup \Sigma_{\bar{h}}^{\#}$. 
Step 2. (Lipschitz partial regularity) By applying [13, Lemma 3] as purely geometric consequence of the inner ball condition established in the previous step, we get the following property: For any $z_{0} \in \Gamma_{\bar{h}}^{\#} \cup \Sigma_{\bar{h}}^{\#}$ there exist an orthonormal basis $\mathbf{i}, \mathbf{j} \in \mathbb{R}^{2}$ and a rectangle

$$
Q:=\left\{z_{0}+s \mathbf{i}+t \mathbf{j}:-a^{\prime}<s<a^{\prime},-b^{\prime}<t<b^{\prime}\right\},
$$

with $a^{\prime}, b^{\prime}>0$, such that $\Omega_{\bar{h}} \cap Q$ has one of the following two representations:

(a) There exists a Lipschitz function $f:\left(-a^{\prime}, a^{\prime}\right) \rightarrow\left(-b^{\prime}, b^{\prime}\right)$ such that $f(0)=0$ and

$$
\Omega_{\bar{h}} \cap Q=\left\{z_{0}+s \mathbf{i}+t \mathbf{j}:-a^{\prime}<s<a^{\prime},-b^{\prime}<t<f(s)\right\} \cap((0, \ell) \times \mathbb{R}) .
$$

Moreover, the function $f$ admits at every point left and right derivatives, which are left and right continuous, respectively.

(b) There exist two Lipschitz functions $f_{1}, f_{2}:\left[0, a^{\prime}\right) \rightarrow\left(-b^{\prime}, b^{\prime}\right)$ such that $f_{i}(0)=\left(f_{i}\right)_{+}^{\prime}(0)=0$ for $i=1,2$, $f_{1} \leq f_{2}$, and

$$
\Omega_{\bar{h}} \cap Q=\left\{z_{0}+s \mathbf{i}+t \mathbf{j}: 0<s<a^{\prime},-b^{\prime}<t<f_{1}(s) \text { or } f_{2}(s)<t<b^{\prime}\right\} .
$$

Moreover, the functions $f_{1}, f_{2}$ admit at every point left and right derivatives, which are left and right continuous, respectively. Note that (a) and (b) imply statement (i) of the theorem and the fact that

$$
\lim _{x \rightarrow x_{0}^{ \pm}} \bar{h}_{ \pm}^{\prime}(x)= \pm \infty \quad \text { for every } x_{0} \text { s.t. }\left(x_{0}, \bar{h}\left(x_{0}\right)\right) \in \Sigma_{\bar{h}}^{\#} \cup \Sigma_{\bar{h}, c}^{\#} .
$$

Step 3. ( $C^{1}$ regularity) By the existence and the continuity properties of right and left derivatives established in the previous step, the $C^{1}$ regularity away from $\Sigma_{\bar{h}}^{\#} \cup \Sigma_{\bar{h}, c}^{\#}$ follows once we show that there cannot be corner points. To this aim one argues by contradiction: Assume that $z_{0}=\left(x_{0}, \bar{h}\left(x_{0}\right)\right)$, with $\bar{h}\left(x_{0}\right)>0$, is a corner point, i.e. $\bar{h}_{-}^{\prime}\left(x_{0}\right) \neq \bar{h}_{+}^{\prime}\left(x_{0}\right)$. Without loss of generality (upon changing the reference frame, if needed) we may also assume that both right and left derivatives are finite. We now implement a blow-up argument. For any $r>0$ we set

$$
A_{r}:=\frac{1}{r}\left[B_{1}(0) \cap\left(\Omega_{\bar{h}}-z_{0}\right)\right]
$$

and note that $\bar{A}_{r} \rightarrow \bar{A}_{0}$ in the Hausdorff sense, where

$$
A_{0}:=\left\{(x, y): y<h_{0}(x)\right\},
$$

with $h_{0}(x)=\bar{h}_{-}^{\prime}\left(x_{0}\right) x$ for $x<0$ and $h_{\infty}(x):=\bar{h}_{+}^{\prime}\left(x_{0}\right) x$ fro $x \geq 0$. We rescale also the function $\bar{u}$ by setting for $z \in A_{r}$

$$
u_{r}(z):=\frac{\bar{u}\left(z_{0}+r z\right)-a_{r}}{\lambda_{r} r}
$$

where

$$
a_{r}:=f_{B_{r}\left(z_{0}\right) \cap \Omega_{\bar{h}}} \bar{u} d z, \quad \lambda_{r}^{2}:=f_{B_{r}\left(z_{0}\right) \cap \Omega_{\bar{h}}}|\nabla \bar{u}|^{2} d z
$$

so that

$$
f_{A_{r}}\left|\nabla u_{r}\right|^{2} d z=1, \quad \int_{A_{r}} u_{r} d z=0 .
$$

At this point, using standard arguments, one can show that any limit function $u_{0}$ of $\left\{u_{r}\right\}$ as $r \rightarrow 0^{+}$is a solution to the Lamé system in $A_{0}$, with natural Neumann conditions on $\partial A_{0} \cap B_{1}(0)$. By a result due to Grisvard ([30]) on the behavior of the solutions of the Lamé system in corner domains, there exists $\alpha>1$ such that

$$
\int_{A_{0} \cap B_{r}(0)}\left|\nabla u_{0}\right|^{2} d z \leq C r^{\alpha} .
$$

In turn, by the blow up proecedure one can show that essentially the same decay rate is "inherited" by $u_{r}$ on $A_{r}$, and this yields the following decay estimate for $\bar{u}$ : For any $\beta \in(1, \alpha)$ we have

$$
\int_{\Omega_{\bar{h}} \cap B_{r}\left(z_{0}\right)}|\nabla \bar{u}|^{2} d z \leq C r^{\beta}
$$


for all $r$ sufficiently small (and with $C$ depending on $\beta$ ). Then one can extend $\bar{u}$ to the whole ball $B_{r_{0}}\left(z_{0}\right)$ in such a way that the resulting function (still denoted by $\bar{u}$ ) satisfies for all $r$ sufficiently small

$$
\int_{B_{r}\left(z_{0}\right)}|\nabla \bar{u}|^{2} d z \leq C r^{\beta}
$$

for some $C$ independent of $r$.

Given $r$ sufficiently small, let $z_{1}\left(x_{1}, y_{1}\right), z_{2}\left(x_{2}, y_{2}\right) \in \Gamma_{\bar{h}}^{\#} \cap \partial B_{r}\left(z_{0}\right)$ two points such that $x_{1}<x_{0}<x_{2}$ and $\Gamma_{\bar{h}}^{\#} \cap\left(\left(x_{1}, x_{2}\right) \times \mathbb{R}\right) \subset B_{r}\left(z_{0}\right)$. We may now define a competing configuration by cutting out the corner. To be precise, we consider $(\tilde{h}, \bar{u})$, where $\tilde{h}$ is the function coinciding with $\bar{h}$ in $[0, \ell) \backslash\left[x_{1}, x_{2}\right]$ and defined in $\left(x_{1}, x_{2}\right)$ as the affine function whose graph is the segment connecting $z_{1}$ and $z_{2}$. Comparing the energies of the two pairs $(\bar{h}, \bar{u}),(\tilde{h}, \bar{u})$ one gets from $(3.2)$ and the fact that $\beta>1$

$$
\left[F(\bar{h}, \bar{u})+\Lambda|| \Omega_{\bar{h}}|-d|\right]-\left[F(\tilde{h}, \bar{u})+\Lambda|| \Omega_{\tilde{h}}|-d|\right] \geq 2 r\left(1-\sin \left(\vartheta_{0} / 2\right)\right)+o(r)>0,
$$

for $r$ small enough, where $\vartheta_{0}$ is the angle (by contradiction assumption different from $\pi$ ) at the corner point $z_{0}$. The last inequality clearly contradicts the minimality of $(\bar{h}, \bar{u})$ established in Step 1.

If the corner point $z_{0}$ lies at the interface with the substrate, i.e., $z_{0}=\left(x_{0}, 0\right)$, then the argument is more delicate: we refer to [15] for the details.

Step 4. (Higher regularity) The $C^{1, \alpha}$ regularity asserted in statement (iii) of Theorem 3.1 can be established by refining the arguments of the previous step. With the $C^{1, \alpha}$ regularity at hand, the analytic regularity follows from the general theory developed in [35].

\section{Second Variation and Minimality}

In this section we establish some relevant local and global minimality results. The main tool will be the study of the second variation of the energy functional $F$.

To this aim, let $(h, u) \in X\left(e_{0} ; \ell\right)$ be an admissible configuration, with $h \in C_{\#}^{\infty}(0, \ell), h>0$ and $u$ the elastic equilibrium corresponding to $\Omega_{h}$, i.e., $u=u_{h}$ (see (2.6)). Given $\psi \in C_{\#}^{\infty}(0, \ell)$ with $\int_{0}^{\ell} \psi d x=0$, for $t \in \mathbb{R}$ let $h_{t}:=h+t \psi$. We set for simplicity $u_{t}:=u_{h_{t}}$, and we define the first and the second variation of $F$ at $(h, u)$ along the direction $\psi$, denoted by $\partial F(h, u)[\psi]$ and $\partial^{2} F(h, u)[\psi]$ respectively, as

$$
\partial F(h, u)[\psi]:=\left.\frac{d}{d t} F\left(h_{t}, u_{t}\right)\right|_{t=0} \quad \text { and } \quad \partial^{2} F(h, u)[\psi]:=\left.\frac{d^{2}}{d t^{2}} F\left(h_{t}, u_{t}\right)\right|_{t=0} .
$$

The first and the second variations can be computed explicitly. Before giving the formula we recall that given $h$

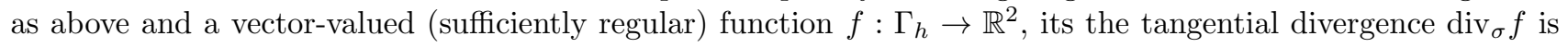
defined by

$$
\operatorname{div}_{\sigma} f:=f_{\sigma} \cdot \sigma,
$$

where $\sigma$ stands for tangential unit vector

$$
\sigma:=\frac{\left(1, h^{\prime}\right)}{\sqrt{1+\left(h^{\prime}\right)^{2}}}
$$

and $(\cdot)_{\sigma}$ for the corresponding tangential derivative. In the case of a matrix-valued function the tangential divergence is computed row by row. We will also denote by $\nu$ the outer unit normal to $\Omega_{h}$ at $\Gamma_{h}$, i.e.,

$$
\nu:=\frac{\left(-h^{\prime}, 1\right)}{\sqrt{1+\left(h^{\prime}\right)^{2}}}
$$

Moreover, the curvature $k$ of $\Gamma_{h}$ isdefined as

$$
k=-\frac{h^{\prime \prime}}{\left(1+\left(h^{\prime}\right)^{2}\right)^{\frac{3}{2}}} .
$$

Finally we set

$$
A\left(\Omega_{h}\right):=\left\{w \in L D_{\#}\left(\Omega_{h} ; \mathbb{R}^{2}\right): w(\cdot, 0) \equiv 0\right\} .
$$


Theorem 4.1 (First and second variation) Let $(h, u), \psi$, and $\left(h_{t}, u_{t}\right)$ be as above, let $\pi_{1}: \mathbb{R}^{2} \rightarrow \mathbb{R}^{\text {be }}$ the orthogonal projection on the $x$-axis, and let $\varphi \in \widetilde{H}_{\#}^{1}\left(\Gamma_{h}\right)$ be defined as $\varphi:=\frac{\psi}{\sqrt{1+h^{\prime 2}}} \circ \pi_{1}$. Then the first variation of $F$ at $(h, u)$ along the direction $\psi$ is given by

$$
\partial F(h, u)[\psi]=\int_{\Gamma_{h}}(Q(E(u))+k) \varphi d \mathcal{H}^{1}
$$

Moreover, the second variation of $F$ at $(h, u)$ along the direction $\psi$ is given by

$$
\begin{aligned}
& \partial^{2} F(h, u)[\psi]= \\
& =-2 \int_{\Omega_{h}} Q\left(E\left(v_{\varphi}\right)\right) d z+\int_{\Gamma_{h}}\left(\varphi_{\sigma}\right)^{2} d \mathcal{H}^{1}+\int_{\Gamma_{h}}\left(\partial_{\nu}[Q(E(u))]-k^{2}\right) \varphi^{2} d \mathcal{H}^{1} \\
& \quad-\int_{\Gamma_{h}}(Q(E(u))+k)\left(\left(h^{\prime} \circ \pi_{1}\right) \varphi^{2}\right)_{\sigma} d \mathcal{H}^{1},
\end{aligned}
$$

where $v_{\varphi} \in A\left(\Omega_{h}\right)$ is the solution to

$$
\int_{\Omega_{h}} \mathbb{C} E\left(v_{\varphi}\right): E(w) d z=\int_{\Gamma_{h}} \operatorname{div}_{\sigma}(\varphi \mathbb{C} E(u)) \cdot w d \mathcal{H}^{1} \quad \text { for all } w \in A\left(\Omega_{h}\right) .
$$

We refer to the proof of [27, Theorem 3.2] for the calculations leading to the above formulas.

Remark 4.2 (Critical configurations) From (4.3) one can infer that a regular configuration ( $h, u)$ as above is critical with respect to volume preserving variations, i.e., $\partial F(h, u)[\psi]=0$ for all $\psi \in C_{\#}^{\infty}(0, \ell)$ with $\int_{0}^{\ell} \psi d x=0$ if and only if

$$
Q(E(u))+k=\lambda \text { on } \Gamma_{h} \text { for some } \lambda \in \mathbb{R}
$$

The constant $\lambda$ is the Lagrange multiplier associated with the volume constraint. In what follows by a regular critical configuration $(h, u)$ we mean a regular configuration as above satisfying (4.6).

Remark 4.3 (Second variation at critical configurations) Let $(h, u)$ and $\varphi$ be as in Theorem 4.1 and assume further that $(h, u)$ is critical. By (4.6),

$$
\int_{\Gamma_{h}}(Q(E(u))+k)\left(\left(h^{\prime} \circ \pi_{1}\right) \varphi^{2}\right)_{\sigma} d \mathcal{H}^{1}=\lambda \int_{\Gamma_{h}}\left(\left(h^{\prime} \circ \pi_{1}\right) \varphi^{2}\right)_{\sigma} d \mathcal{H}^{1}=0,
$$

where the last equality follows from the periodicity of the functions involved. Therefore, the second variation at critical configurations reduces to

$$
\begin{aligned}
\partial^{2} F(h, u)[\psi]=-2 \int_{\Omega_{h}} Q\left(E\left(v_{\varphi}\right)\right) d z & +\int_{\Gamma_{h}}\left(\varphi_{\sigma}\right)^{2} d \mathcal{H}^{1} \\
& +\int_{\Gamma_{h}}\left(\partial_{\nu}[Q(E(u))]-k^{2}\right) \varphi^{2} d \mathcal{H}^{1}=: Q(h, u)[\psi]
\end{aligned}
$$

Finally, notice that the above quadratic form $Q(h, u)[\cdot]$ is well defined at any $\psi \in H_{\#}^{1}(0, \ell)\left(\right.$ with $\left.\int_{0}^{\ell} \psi d x=0\right)$.

The main result of the subsection is represented by the following local minimality criterion.

Theorem 4.4 (Local minimality criterion) Let $(h, u) \in X\left(e_{0} ; \ell\right)$ be a regular critical configuration for $F$ as in Remark 4.2, and assume that

$$
\partial^{2} F(h, u)[\psi]>0 \quad \text { for all } \psi \in H_{\#}^{1}(0, \ell) \backslash\{0\}, \text { with } \int_{0}^{\ell} \psi d x=0,
$$

i.e., the second variation of $F$ at $(h, u)$ is positive definite with respect to volume preserving variations. Then $(h, u)$ is an isolated local minimizer with respect to $L^{\infty}$-small perturbations of the profile $h$, i.e., there exists $\delta>0$ such that

$$
F(h, u)<F(g, v)
$$

for all $(g, v) \in X\left(e_{0} ; \ell\right)$ with $\left|\Omega_{g}\right|=\left|\Omega_{h}\right|$ and $0<\sup _{[0, \ell]}|g-h| \leq \delta$. 


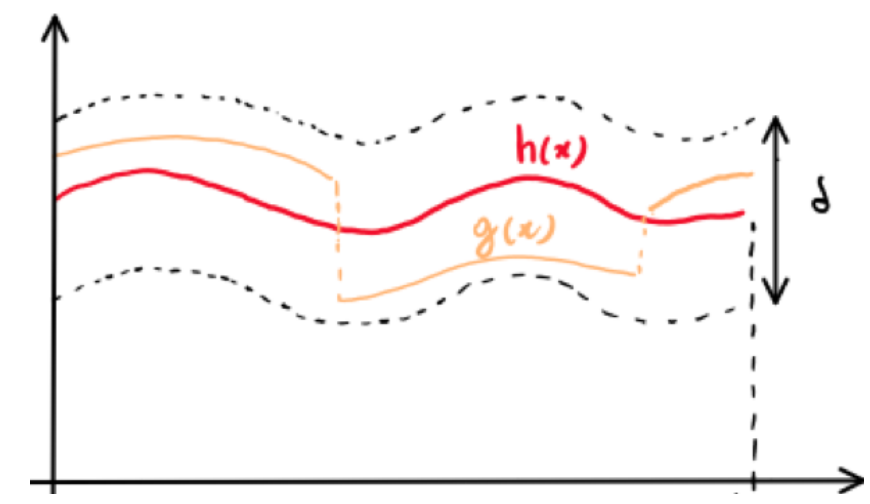

Figure 4.1: A locally minimizing profile $h$ and an admissible competitor $g$

The proof of the theorem is very long and technical and we refer to [27] for its proof. Below we outline the overall strategy and the main steps of the argument.

Outline of the proof of Theorem 4.4. We divide the outline into several steps.

Step 1. (Coercivity of the quadratic form at $(h, u)$ ) A compactness argument shows that (4.8) is equivalent to the following coercivity: There exists $c_{0}>0$ such that

$$
\partial^{2} F(h, u)[\psi]=Q(h, u)[\psi] \geq c_{0}\|\psi\|_{H^{1}(0, \ell)}^{2}
$$

for all $\psi \in H_{\#}^{1}(0, \ell) \backslash\{0\}$, with $\int_{0}^{\ell} \psi d x=0$. We recall that $Q(h, u)[\cdot]$ is the quadratic form defined in (4.7).

Step 2. (Coercivity of the quadratic form at $W^{2, \infty}$-close configurations) Here one shows that there exists $\delta_{0}>0$ with the following property: If $\left(g, u_{g}\right) \in X\left(e_{0}, \ell\right)$ is such that $g \in W_{\#}^{2, \infty}(0, \ell),\left|\Omega_{g}\right|=\left|\Omega_{h}\right|$ and $0<\|g-h\|_{W_{\#}^{2, \infty}(0, \ell)} \leq \delta_{0}$, then

$$
Q\left(g, u_{g}\right)[\psi] \geq \frac{c_{0}}{2}\|\psi\|_{H^{1}(0, \ell)}^{2}
$$

for all $\psi \in H_{\#}^{1}(0, \ell) \backslash\{0\}$, with $\int_{0}^{\ell} \psi d x=0$, where $c_{0}$ is the constant introduced in Step 1 . This is perhaps the most technical part of the proof and amounts to show the continuity of the first eigenvalue of the quadratic form $Q\left(g, u_{g}\right)$ with respect to the $W^{2, \infty}$-convergence of $g$. Since the explicit formula of $Q$ involves also the trace of the gradient of $E\left(u_{g}\right)$ on $\Gamma_{g}$, this analysis requires rather delicate regularity estimates in the appropriate fractional Sobolev spaces.

Step 3. ( $W^{2, \infty}$-local minimality) The property established in Step 2 easily leads to the following preliminary minimality result: There exists $\delta_{1} \in\left(0, \delta_{0}\right)$ such that:

$$
\begin{aligned}
g \in W_{\#}^{2, \infty}(0, \ell),\left|\Omega_{g}\right|=\left|\Omega_{h}\right|, \text { and } 0 & <\|g-h\|_{W^{2, \infty}(0, \ell)} \leq \delta_{1} \\
& \Longrightarrow F(h, u)<F\left(g, u_{g}\right) .
\end{aligned}
$$

Let $\lambda$ be the Lagrange multiplier associate with the critical configuration $(h, u)$ (see (4.6)), and observe first that there exists a modulus of continuity $\omega$ with the following property:

$$
g \in W_{\#}^{2, \infty}(0, \ell) \text { and }\|g-h\|_{W^{2, \infty}(0, \ell)} \leq \delta \Longrightarrow\left\|Q\left(E\left(u_{g}\right)\right)+k_{g}-\lambda\right\|_{L^{\infty}\left(\Gamma_{g}\right)} \leq \omega(\delta),
$$

where $\omega(\delta) \rightarrow 0$ as $\delta \rightarrow 0^{+}$. Here we write $k_{g}$ (instead of $k$ ) to denote the curvature of $\Gamma_{g}$. The above property follows from standard elliptic estimates on the solution $u_{g}$ of the Lamé system. In turn, if $\|g-h\|_{W_{\#}^{2, \infty}(0, \ell)} \leq \delta$, with $\delta \in\left(0, \delta_{0}\right)$, and $\psi$ is as in Theorem 4.1, then we obtain

$$
\begin{aligned}
\int_{\Gamma_{g}}\left(Q\left(E\left(u_{g}\right)\right)+k_{g}\right)\left(\left(g^{\prime} \circ \pi_{1}\right) \varphi^{2}\right)_{\sigma} d \mathcal{H}^{1} & =\int_{\Gamma_{g}}\left(Q\left(E\left(u_{g}\right)\right)+k_{g}-\lambda\right)\left(\left(g^{\prime} \circ \pi_{1}\right) \varphi^{2}\right)_{\sigma} d \mathcal{H}^{1} \\
& \geq-C\left\|Q\left(E\left(u_{g}\right)\right)+k_{g}-\lambda\right\|_{L^{\infty}\left(\Gamma_{g}\right)}\|\varphi\|_{H^{1}\left(\Gamma_{g}\right)}^{2} \\
& \geq-C \omega(\delta)\|\psi\|_{H^{1}(0, \ell)}^{2},
\end{aligned}
$$


where $C>0$ is a constant which may change from line to line and depends only on $\delta_{0}$, and $\varphi:=\frac{\psi}{\sqrt{1+g^{\prime 2}}} \circ \pi_{1}$. Let now $g$ be as in (4.9) and set $h_{t}:=h+t(g-h)$ and $f(t):=F\left(h_{t}, u_{h_{t}}\right)$, so that $F(h, u)=f(0)$ and $F\left(g, u_{g}\right)=f(1)$. Note also that since $(h, u)$ is critical we have $f^{\prime}(0)=0$. Moreover, by Theorem 4.1 and $(4.7)$, setting $\psi:=g-h$ and letting $\varphi_{t}:=\frac{\psi}{\sqrt{1+h_{t}^{\prime 2}}} \circ \pi_{1}$, we have

$$
f^{\prime \prime}(t)=Q\left(h_{t}, u_{h_{t}}\right)[\psi]+\int_{\Gamma_{h_{t}}}\left(Q\left(E\left(u_{h_{t}}\right)\right)+k_{h_{t}}\right)\left(\left(h_{t}^{\prime} \circ \pi_{1}\right) \varphi_{t}^{2}\right)_{\sigma} d \mathcal{H}^{1}
$$

Thus, also using Step 2 and (4.10) (with $h_{t}$ in place of $g$ ), we have

$$
\begin{aligned}
F(h, u) & =f(0)=f(1)-\int_{0}^{1}(1-t) f^{\prime \prime}(t) d t \\
& <f(1)-\left(\frac{c_{0}}{2}-C \omega\left(\delta_{1}\right)\right)\|\psi\|_{H^{1}(0, \ell)}^{2} \int_{0}^{1}(1-t) d t \\
& =F\left(g, u_{g}\right)-\left(\frac{c_{0}}{4}-\frac{C}{2} \omega\left(\delta_{1}\right)\right)\|\psi\|_{H^{1}(0, \ell)}^{2} .
\end{aligned}
$$

Claim (4.9) follows by choosing $\delta_{1}$ so small that $\frac{c_{0}}{2}-C \omega\left(\delta_{1}\right)>0$.

Step 4. ( $W^{2, \infty}$-local minimality implies $L^{\infty}$-minimality) In this step one shows that the $W^{2, \infty}$-local minimality established in the previous step is in fact equivalent to the local minimality with respect to any admissible (possibly irregular) profile sufficiently close in the sup norm, thus proving the theorem. The argument goes as follows: Assume by contradiction that the $W^{2, \infty}$-local minimizer $(h, u)$ is not a local minimizer. Then one can find a sequence of configurations $\left(k_{n}, w_{n}\right)$ with $0<\sup _{[0, \ell]}\left|h-k_{n}\right| \leq \frac{1}{n},\left|\Omega_{k_{n}}\right|=\left|\Omega_{h}\right|$, and $F\left(k_{n}, w_{n}\right) \leq$ $F(h, u)$. Consider the obstacle problems

$$
\min \left\{F(g, v)+\Lambda|| \Omega_{g}|-| \Omega_{h}||:(g, v) \in X\left(e_{0}, \ell\right), g \geq h-\frac{1}{n}\right\}
$$

with $\Lambda>0$, and let $\left(g_{n}, v_{n}\right)$ be the corresponding minimizing configurations. Notice that we have replaced the volume constraint by a penalization term. Since $\left(k_{n}, w_{n}\right)$ is an admissible competitor for (4.11), we have in particular

$$
F\left(g_{n}, v_{n}\right) \leq F\left(g_{n}, v_{n}\right)+\Lambda|| \Omega_{g_{n}}|-| \Omega_{h}|| \leq F\left(k_{n}, w_{n}\right) \leq F(h, u) .
$$

We want to show that if $\Lambda$ is sufficiently large, then $g_{n}$ is of class $W^{2, \infty}$ and $g_{n} \rightarrow h$ in $W^{2, \infty}$.

To this aim, one first proves that if $\Lambda>\left\|k_{h}\right\|_{L^{\infty}}$ (where $k_{h}$ denotes as before the curvature of $\Gamma_{h}$ ), then $(h, u)$ is the unique minimizer to

$$
\min \left\{F(g, v)+\Lambda|| \Omega_{g}|-| \Omega_{h}||:(g, v) \in X\left(e_{0}, \ell\right), g \geq h\right\}
$$

This follows easily from the estimate

$$
\mathcal{H}^{1}\left(\Gamma_{g}\right)+\left\|k_{h}\right\|_{L^{\infty}} \int_{0}^{\ell}|g-h| d x \geq \mathcal{H}^{1}\left(\Gamma_{h}\right)
$$

for all $g \in A P(0, \ell)$, which in turn can be shown arguing similarly to the proof of Lemma 3.4. Since $(h, u)$ is the unique minimizer of $(4.13)$, we easily deduce that $\left(g_{n}, v_{n}\right)$ must converge to $(h, u)$. In particular,

$$
g_{n} \rightarrow h \quad \text { in } L^{\infty}(0, \ell)
$$

Next, we observe that from the definition of $F$ the profile $g_{n}$ minimizes the functional

$$
g \rightarrow \mathcal{H}^{1}\left(\Gamma_{g}\right)+2 \mathcal{H}^{1}\left(\Sigma_{g}\right)+\Lambda|| \Omega_{g}|-| \Omega_{h}||
$$

among all admissible $g$ such that $h-\frac{1}{n} \leq g \leq g_{n}$. This one-sided minimality property yields a uniform inner ball condition at all points of $\Gamma_{g_{n}} \cup \Sigma_{g_{n}}$. To be precise, arguing as in Lemma 3.4 we show that for all $z \in \Gamma_{g_{n}} \cup \Sigma_{g_{n}}$ there exists a ball $B_{\varrho_{0}}\left(z_{0}\right) \subset \Omega_{g_{n}}^{\#}$ such that $z \in \partial B_{\varrho_{0}}\left(z_{0}\right)$, with $\varrho_{0}$ independent of $n$.

As a purely geometric consequence of this uniform inner ball condition and of (4.14), we can deduce that $g_{n}$ has no cusps nor vertical cuts for $n$ large. The heuristic idea is the following: If $\Gamma_{g_{n}}$ contains a cusp or a 


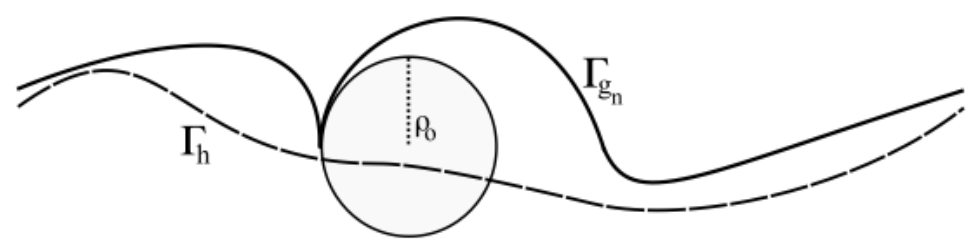

Figure 4.2: If $\Gamma_{g_{n}}$ contains a cusp (or a vertical crack), then the $L^{\infty}$-distance between $g_{n}$ and $h$ is comparable to $\rho_{0}$.

vertical crack, then, due to the inner ball condition, the $L^{\infty}$-distance between $g_{n}$ and $h$ is comparable to $\rho_{0}$, a contradiction to (4.14) (see Figure 4.2). A close inspection of this argument shows that, in fact, $g_{n} \rightarrow h$ in $C^{1}([0, \ell])$.

In view of this convergence, by a blow-up argument similar to the one outilined in the proof of Theorem 3.1 we obtain the following decay estimate for the gradients of the displacements $v_{n}$ :

$$
\int_{B_{r}(z) \cap \Omega_{g_{n}}}\left|\nabla v_{n}\right|^{2} d w \leq C_{0} r^{2-\delta}
$$

for all $z \in \Gamma_{g_{n}}, r \in\left(0, r_{0}\right)$, where $C_{0}$ and $r_{0}$ are independent of $n$ and $\delta$ is any fixed small positive constant.

With this estimate at hand, the deviation from flatness of $\Gamma_{g_{n}}$ can be estimated by comparing the energy of $\left(g_{n}, v_{n}\right)$ in $B_{r}(z) \cap \Omega_{g_{n}}$ with that of the modified configuration obtained by extending $v_{n}$ to the whole ball and by replacing $\Gamma_{g_{n}} \cap B_{r}(z)$ with the segments connecting the center $z$ to the points in $\Gamma_{g_{n}} \cap \partial B_{r}(z)$. The comparison argument yields an estimate of the oscillation of the unit normal vectors to $\Gamma_{g_{n}}$ that implies a uniform bound of the $C^{1, \alpha}$-norms of $\left\{g_{n}\right\}$ for $\alpha \in\left(0, \frac{1}{2}\right)$. In turn, by elliptic regularity, we deduce that also $\left\{v_{n}\right\}$ is uniformly bounded in the $C^{1, \alpha}$-norm.

The $C^{1, \alpha}$-bound allows one to conclude that $\left(g_{n}, v_{n}\right)$ satisfy the Euler-Lagrange equation (4.6) in a weak sense, namely, for every $n \in \mathbb{N}$ sufficiently large

$$
Q\left(E\left(v_{n}\right)\right)+k_{g_{n}}=\lambda_{n} \quad \text { on } \Gamma_{g_{n}} \backslash \Gamma_{h-\frac{1}{n}},
$$

for a suitable constant Lagrange-multiplier $\lambda_{n}$. In turn, it follows from the above identity that in fact $g_{n}$ is of class $W^{2, \infty}$. Moreover, by an integration by parts argument, the $C^{1, \alpha}$-convergence of $g_{n}$ to $h$ yields also that $\lambda_{n} \rightarrow \lambda$, with $\lambda$ the Lagrange multiplier associated with $h$. With this last piece of information at hand, (4.15) implies that in fact $g_{n} \rightarrow h$ in $W^{2, \infty}$.

In turn, by the isolated $W^{2, \infty}$-local minimality of $(h, u)$ established in the previous step and by (4.12), we must have $g_{n}=h$ for $n$ large enough and $F\left(k_{n}, w_{n}\right)=F\left(g_{n}, v_{n}\right)=F(h, u)$, i.e., $\left(k_{n}, w_{n}\right)$ is itself a minimizer of (4.11). But then, repeating all the above argument with $\left(g_{n}, v_{n}\right)$ replaced by $\left(k_{n}, w_{n}\right)$ we arrive at the conclusion that $k_{n}=h$ for $n$ large enough, a contradiction to the fact that $\sup _{[0, \ell]}\left|h-k_{n}\right|>0$ for all $n$.

We now apply Therorem 4.4 to study the local and global minimality of the flat configuration. To this aim, given $d>0$, the pair $\left(h, u_{e_{0}}\right) \in X\left(e_{0} ; \ell\right)$ defined as

$$
h \equiv \frac{d}{\ell}, \quad u_{e_{0}}(x, y):=\left(e_{0} x, \frac{-\lambda e_{0}}{2 \mu+\lambda} y\right),
$$

will be referred to as the flat configuration with volume $d$. With a slight abuse of notation, we simply write $\left(d / \ell, u_{e_{0}}\right)$ to denote such a configuration. Note that $\left(d / \ell, u_{e_{0}}\right)$ is a critical configuration; indeed, it can be checked that $u_{e_{0}}$ is the elastic equilibrium corresponding to any flat profile, and clearly (4.6) is satisfied.

The results below provide an analytical validation of the experimental evidence that when the thickness of the film is sufficiently small the flat configuration is a minimizer. In the first theorem below we analytically determine the local minimality threshold of the flat configuration. This will be done in terms of the Grinfeld 
function $K$ (see [29]) defined for $y \geq 0$ by

$$
K(y):=\max _{n \in \mathbb{N}} \frac{1}{n} J(n y), \quad \text { where } J(y):=\frac{y+\left(3-4 \nu_{p}\right) \sinh y \cosh y}{4\left(1-\nu_{p}\right)^{2}+y^{2}+\left(3-4 \nu_{p}\right) \sinh ^{2} y},
$$

$\nu_{p}$ being the Poisson modulus of the elastic material, i.e.,

$$
\nu_{p}:=\frac{\lambda}{2(\lambda+\mu)} .
$$

One can show (see [27, Corollary 5.3]) that,

$K$ is strictly increasing and continuous, $K(y) \leq C y$, and $\lim _{y \rightarrow+\infty} K(y)=1$,

for some positive constant $C$.

Theorem 4.5 (Local minimality of the flat configuration) Let $d_{\mathrm{loc}}:(0,+\infty) \rightarrow(0,+\infty]$ be defined as $d_{\mathrm{loc}}(\ell):=+\infty$, if $0<\ell \leq \frac{\pi}{4} \frac{2 \mu+\lambda}{e_{0}^{2} \mu(\mu+\lambda)}$, and as the solution to

$$
K\left(\frac{2 \pi d_{\mathrm{loc}}(\ell)}{\ell^{2}}\right)=\frac{\pi}{4} \frac{2 \mu+\lambda}{e_{0}^{2} \mu(\mu+\lambda)} \frac{1}{\ell},
$$

otherwise. If $0<d<d_{\mathrm{loc}}(\ell)$, then the flat configuration $\left(d / \ell, u_{e_{0}}\right)$ is an isolated local minimizer for $F$ with respect to perturbations of the profile sufficiently small in the sup-norm, i.e., in the sense stated in Theorem 4.4.

Moreover, the threshold $d_{\mathrm{loc}}$ is critical: Indeed, for $d>d_{\mathrm{loc}}(\ell)$ there exists $(g, v) \in X\left(e_{0} ; \ell\right)$, with $\left|\Omega_{g}\right|=d$, and $\sup _{[0, \ell]}|g-d / \ell|$ arbitrarily small such that $F(g, v)<F\left(d / \ell, u_{e_{0}}\right)$.

Proof. By Theorem 4.4 it is enough to show that

$$
\begin{aligned}
\partial^{2} F\left(\frac{d}{\ell}, u_{e_{0}}\right)[\psi]>0 \quad \text { for all } \psi \in H_{\#}^{1}(0, \ell) \backslash\{0\}, & \int_{0}^{\ell} \psi d x=0 \\
& \Longleftrightarrow K\left(\frac{2 \pi d}{\ell^{2}}\right)<\frac{\pi}{4} \frac{2 \mu+\lambda}{e_{0}^{2} \mu(\mu+\lambda)} \frac{1}{\ell}
\end{aligned}
$$

and, conversely,

$$
\begin{aligned}
K\left(\frac{2 \pi d}{\ell^{2}}\right)>\frac{\pi}{4} \frac{2 \mu+\lambda}{e_{0}^{2} \mu(\mu+\lambda)} & \frac{1}{\ell} \\
& \Longrightarrow \partial^{2} F\left(\frac{d}{\ell}, u_{e_{0}}\right)[\psi]<0 \quad \text { for some admissible } \psi .
\end{aligned}
$$

Let us write (4.7) for the flat configuration. To this aim, recalling (4.16), observe that

$$
\mathbb{C} E\left(u_{e_{0}}\right)=\left(\begin{array}{ll}
\sigma_{0} & 0 \\
0 & 0
\end{array}\right), \text { where } \sigma_{0}:=e_{0} \frac{4 \mu(\mu+\lambda)}{2 \mu+\lambda} .
$$

Let $\psi$ be an admissible variation as in (4.20). Observing that $\operatorname{div}_{\sigma}\left(\mathbb{C} E\left(u_{e_{0}}\right) \psi\right)=\operatorname{div}\left(\mathbb{C} E\left(u_{e_{0}}\right) \psi\right)=\sigma_{0}\left(\psi^{\prime}, 0\right)$, we get

$$
\partial^{2} F\left(\frac{d}{\ell}, u_{e_{0}}\right)[\psi]=-2 \int_{\Omega_{h}} Q\left(E\left(v_{\psi}\right)\right) d z+\int_{0}^{\ell} \psi^{\prime 2} d x
$$

where $h \equiv d / \ell, \Omega_{h}=(0, \ell) \times(0, d / \ell)$ and $v_{\psi} \in A\left(\Omega_{h}\right)$ is the unique solution to the equation

$$
\int_{\Omega_{h}} \mathbb{C} E\left(v_{\psi}\right): E(w) d z=\sigma_{0} \int_{0}^{\ell} w_{1}(x, d / \ell) \psi^{\prime}(x) d x
$$

for all $w=\left(w_{1}, w_{2}\right) \in A\left(\Omega_{h}\right)$. We perform the change of variables

$$
\tilde{v}_{\psi}(x, y):=\frac{2 \pi}{\ell} v_{\psi}\left(\frac{\ell}{2 \pi} x, \frac{\ell}{2 \pi} y\right), \quad \tilde{\psi}(x):=\frac{2 \pi}{\ell} \psi\left(\frac{\ell}{2 \pi} x\right)
$$


and we observe that $\tilde{v}_{\psi}$ satisfies (4.24) in the interval $(0,2 \pi)$, with $\psi$ replaced by $\tilde{\psi}$. Under the above change of variables and setting $\tilde{h}(x) \equiv 2 \pi d / \ell^{2},(4.23)$ may be rewritten as

$$
\begin{aligned}
\partial^{2} F\left(\frac{d}{\ell}, u_{e_{0}}\right)[\psi] & =\frac{\ell^{2}}{4 \pi^{2}}\left[-2 \int_{\Omega_{\tilde{h}}} Q\left(E\left(\tilde{v}_{\psi}\right)\right) d z+\frac{2 \pi}{\ell} \int_{0}^{2 \pi} \tilde{\psi}^{\prime 2} d x\right] \\
& =\frac{\ell^{2}}{4 \pi^{2}} \int_{0}^{2 \pi}\left[-\sigma_{0} \tilde{v}_{\psi 1}(x, \tilde{h}) \tilde{\psi}^{\prime}(x)+\frac{2 \pi}{\ell} \tilde{\psi}^{\prime 2}\right] d x
\end{aligned}
$$

where the last equality follows from (4.24). Here, and in what follows, we write $\tilde{v}_{\psi}=\left(\tilde{v}_{\psi 1}, \tilde{v}_{\psi 2}\right)$.

In order to compute the second variation, let us now solve equation $(4.24)$ in $\Omega_{\tilde{h}}=(0,2 \pi) \times(0, \tilde{h})$ by considering the expansion in Fourier series of $\tilde{v}_{\psi}(\cdot, y)$ for all $y \in(0, \tilde{h})$. To this aim, we set for all $y$ and $n \in \mathbb{Z}$

$$
a_{n}(y):=\frac{1}{\sqrt{2 \pi}} \int_{0}^{2 \pi} \mathrm{e}^{-i n x} \tilde{v}_{\psi 1}(x, y) d x, \quad b_{n}(y):=\frac{1}{\sqrt{2 \pi}} \int_{0}^{2 \pi} \mathrm{e}^{-i n x} \tilde{v}_{\psi 2}(x, y) d x,
$$

and

$$
\tilde{\psi}_{n}:=\frac{1}{\sqrt{2 \pi}} \int_{0}^{2 \pi} \mathrm{e}^{-i n x} \tilde{\psi}(x) d x .
$$

Recalling (2.2) and (4.22), equation (4.24) reads

$$
\begin{cases}(2 \mu+\lambda) \frac{\partial^{2} \tilde{v}_{\psi 1}}{\partial x^{2}}+\mu \frac{\partial^{2} \tilde{v}_{\psi 1}}{\partial y^{2}}+(\lambda+\mu) \frac{\partial^{2} \tilde{v}_{\psi 2}}{\partial x \partial y}=0 & \text { in } \Omega_{\tilde{h}}, \\ \mu \frac{\partial^{2} \tilde{v}_{\psi 2}}{\partial x^{2}}+(2 \mu+\lambda) \frac{\partial^{2} \tilde{v}_{\psi 2}}{\partial y^{2}}+(\lambda+\mu) \frac{\partial^{2} \tilde{v}_{\psi 1}}{\partial x \partial y}=0 & \text { in } \Omega_{\tilde{h}}, \\ \frac{\partial \tilde{v}_{\psi 1}}{\partial y}+\frac{\partial \tilde{v}_{\psi 2}}{\partial x}=\frac{\sigma_{0}}{\mu} \tilde{\psi}^{\prime}, \lambda \frac{\partial \tilde{v}_{\psi 1}}{\partial x}+(2 \mu+\lambda) \frac{\partial \tilde{v}_{\psi 2}}{\partial y}=0 & \text { on }\{y=\tilde{h}\}, \\ \tilde{v}_{\psi}=0 & \text { on }\{y=0\} .\end{cases}
$$

In turn, the Fourier coefficients $a_{n}, b_{n}$ solve the following system

$$
\begin{cases}\mu a_{n}^{\prime \prime}-i n(\lambda+\mu) b_{n}^{\prime}-(2 \mu+\lambda) n^{2} a_{n}=0 & \text { in }(0, \tilde{h}), \\ (2 \mu+\lambda) b_{n}^{\prime \prime}-i n(\lambda+\mu) a_{n}^{\prime}-\mu n^{2} b_{n}=0 & \text { in }(0, \tilde{h}), \\ a_{n}^{\prime}(\tilde{h})-i n b_{n}(\tilde{h})=-\frac{\sigma_{0}}{\mu} i n \tilde{\psi}_{n},-i n \lambda a_{n}(\tilde{h})+(2 \mu+\lambda) b_{n}^{\prime}(\tilde{h})=0, & \\ a_{n}(0)=b_{n}(0)=0 . & \end{cases}
$$

By (4.25), we obtain

$$
\begin{aligned}
\partial^{2} F\left(\frac{d}{\ell}, u_{e_{0}}\right)[\psi] & =\frac{\ell^{2}}{4 \pi^{2}} \int_{0}^{2 \pi}\left[-\sigma_{0} \tilde{\psi}_{\psi 1}(x, \tilde{h}) \tilde{\psi}^{\prime}(x)+\frac{2 \pi}{\ell} \tilde{\psi}^{\prime 2}\right] d x \\
& =\frac{\ell^{2}}{4 \pi^{2}} \sum_{n \in \mathbb{Z}}\left[-\sigma_{0} i n a_{n}(\tilde{h}) \tilde{\psi}_{-n}+\frac{2 \pi}{\ell} n^{2} \tilde{\psi}_{n} \tilde{\psi}_{-n}\right] \\
& =\frac{\ell}{2 \pi} \sum_{n \in \mathbb{Z}} n^{2} \tilde{\psi}_{n} \tilde{\psi}_{-n}\left[1-\frac{\sigma_{0}^{2}\left(1-\nu_{p}\right) b J(n \tilde{h})}{2 \pi \mu n}\right]
\end{aligned}
$$

where $J$ is the function introduced in (4.17), and where the last equality follows by explicitly solving (4.26). We refer to [27] for the lengthy (but straightforward) computations. Using also (4.18) and (4.22) we have

$$
\sup _{n \in \mathbb{Z}} \frac{\sigma_{0}^{2}\left(1-\nu_{p}\right) b J\left(2 \pi n d / \ell^{2}\right)}{2 \pi \mu n} \gtrless 1 \Longleftrightarrow K\left(\frac{2 \pi d}{\ell^{2}}\right) \gtrless \frac{\pi}{4} \frac{2 \mu+\lambda}{e_{0}^{2} \mu(\mu+\lambda)} \frac{1}{\ell},
$$

and the conclusion follows from (4.27).

Next theorem addresses the global minimality of the flat configurations. It shows that, for sufficiently small values of $d$, the flat configuration is always the unique global minimizer of (2.5). 
Theorem 4.6 (Global minimality of the flat configuration) The following two statements hold.

(i) For every $\ell>0$ there exists $0<d_{\mathrm{glob}}(\ell) \leq d_{\mathrm{loc}}(\ell)$ (see Theorem 4.5) such that the flat configuration $\left(d / \ell, u_{e_{0}}\right)$ is a global minimizer, i.e., a solution to (2.5) if and only $0<d \leq d_{\text {glob }}(\ell)$. Moreover, if $0<d<d_{\text {glob }}(\ell)$, then $\left(d / \ell, u_{e_{0}}\right)$ is the unique global minimizer.

(ii) There exists $0<\ell_{\text {crit }} \leq \frac{\pi}{4} \frac{2 \mu+\lambda}{e_{0}^{2} \mu(\mu+\lambda)}$ such that $d_{\text {glob }}(\ell)=+\infty$ if and only if $0<\ell \leq \ell_{\text {crit }}$, i.e., the flat configuration $\left(d / \ell, u_{e_{0}}\right)$ is the unique solution to (2.5) for all $d>0$ if and only if $0<\ell \leq \ell_{\text {crit }}$.

Proof. We only prove part (i) of the statement, referring to [27] in what concerns (ii). Fix $b>0$. We start by showing that There exists $d_{0}>0$ such that for $0<d<d_{0}$,

$$
\left(d / \ell, u_{e_{0}}\right) \text { is a global minimizer. }
$$

Clearly, $d_{0}$ possibly depends on $\ell$.

In order to prove the claim, we argue by contradiction, by assuming that there exist a sequence $d_{n} \searrow 0$, a sequence $\left(k_{n}, w_{n}\right) \in X\left(e_{0} ; \ell\right)$ minimizing $F$ under the constraint $\left|\Omega_{k_{n}}\right|=d_{n}$, such that $F\left(k_{n}, w_{n}\right)<F\left(d_{n} / \ell, u_{e_{0}}\right)$. Pick $t \in\left(0, d_{\text {loc }}(\ell)\right)$, where $d_{\text {loc }}$ is the local minimality threshold introduced in Theorem 4.5 , so that $\partial^{2} F\left(t, u_{e_{0}}\right)$ is positive definite and the flat configuration $\left(t, u_{e_{0}}\right)$ is a an isolated local minimizer for $F$. We set

$$
t_{n}:=t-\frac{d_{n}}{\ell}
$$

and we denote by $\left(g_{n}, v_{n}\right)$ a sequence of minimizers of the following problems

$$
\min \left\{F(g, v)+\Lambda|| \Omega_{g}|-t \ell|:(g, v) \in X\left(e_{0} ; \ell\right), g \geq t_{n}\right\},
$$

where $\Lambda>Q_{0}, Q_{0}$ being the constant defined in Lemma 3.3. Since $\left(t, u_{e_{0}}\right)$ is obviously the unique global minimizer of

$$
\min \left\{F(g, v)+\Lambda|| \Omega_{g}|-t \ell|:(g, v) \in X\left(e_{0} ; \ell\right), g \geq t\right\}
$$

it can be shown that $g_{n} \rightarrow t$ in $L^{1}(0, \ell)$ and, also, $\lim _{n \rightarrow \infty} \mathcal{H}^{1}\left(\Gamma_{g_{n}} \cup \Sigma_{g_{n}}\right)=\ell$. This, in turn, implies that $\sup _{[0, \ell]}\left|g_{n}-t\right| \rightarrow 0$.

Moreover, arguing as in the proof of Lemma 3.3, we may deduce that $\left|\Omega_{g_{n}}\right| \geq t b$ so that the functions $\tilde{g}_{n}:=$ $g_{n}-\left(\left|\Omega_{g_{n}}\right|-t \ell\right) / \ell$ satisfy $\sup _{[0, \ell]}\left|\tilde{g}_{n}-t\right| \rightarrow 0$ and

$$
F\left(\tilde{g}_{n}, v_{n}\right) \leq F\left(g_{n}, v_{n}\right) .
$$

Setting

$$
\tilde{k}_{n}:=k_{n}+t_{n} \quad \tilde{w}_{n}(x, y):= \begin{cases}\left(e_{0} x, \frac{-\lambda e_{0}}{2 \mu+\lambda} y\right) & \text { if } 0 \leq y \leq t_{n}, \\ w_{n}\left(x, y-t_{n}\right)+\left(0, \frac{-\lambda e_{0}}{2 \mu+\lambda} t_{n}\right) & \text { if } y \geq t_{n},\end{cases}
$$

we have by construction $\left|\Omega_{\tilde{k}_{n}}\right|=t \ell$. Therefore, using (4.30), the minimality of $\left(g_{n}, v_{n}\right)$ in (4.29), and the contradiction assumption, we deduce that

$$
\begin{aligned}
F\left(\tilde{g}_{n}, v_{n}\right) & \leq F\left(g_{n}, v_{n}\right)+\Lambda|| \Omega_{g}|-t b| \\
& \leq F\left(\tilde{k}_{n}, \tilde{w}_{n}\right)=F\left(k_{n}, w_{n}\right)+Q_{e_{0}} t_{n} \ell \\
& <F\left(d_{n} / \ell, u_{e_{0}}\right)+Q_{e_{0}} t_{n} \ell=F\left(t, u_{e_{0}}\right),
\end{aligned}
$$

where we denoted by $Q_{e_{0}}$ the constant value of $Q\left(E\left(u_{e_{0}}\right)\right)$. Since $\sup _{[0, \ell]}\left|\tilde{g}_{n}-t\right| \rightarrow 0$, the inequality above contradicts the local minimality of $t$, thus asserting (4.28).

Now set

$$
d_{\text {glob }}(\ell):=\sup \left\{d>0:\left(\frac{d}{\ell}, u_{e_{0}}\right) \text { is a minimizer of }(2.5) \text { in the interval }(0, \ell)\right\} .
$$

In order to show that for $d \in\left(0, d_{\text {glob }}(\ell)\right)$ the flat configuration is the unique minimizer, it will be sufficient to prove the following fact: 
If for some $d>0$ the minimum problem (2.5) has a non-flat solution, then, for all $d^{\prime}>d$ the flat configuration is not a global minimizer for (2.5), with $d$ replaced by $d^{\prime}$.

Let $d^{\prime}>d$ and let $(g, v)$ be a non flat minimizer for $(2.5)$. We set

$$
\tilde{g}:=g+\frac{d^{\prime}-d}{\ell} \quad \tilde{v}(x, y):= \begin{cases}\left(e_{0} x, \frac{-\lambda e_{0}}{2 \mu+\lambda} y\right) & \text { if } 0 \leq y \leq \frac{d^{\prime}-d}{\ell}, \\ v\left(x, y-\frac{d^{\prime}-d}{\ell}\right)+\left(0, \frac{-\lambda e_{0}}{2 \mu+\lambda} \frac{d^{\prime}-d}{\ell}\right) & \text { if } y \geq \frac{d^{\prime}-d}{\ell} .\end{cases}
$$

Let $\left(d^{\prime} / \ell, u_{e_{0}}\right)$ be the flat configuration and consider the minimizer $u_{\tilde{g}}$ of the elastic energy in $\Omega_{\tilde{g}}$ (see $(2.6)$ ). Note that

$$
\int_{\Omega_{\tilde{g}}} Q\left(E\left(u_{\tilde{g}}\right)\right) d z<\int_{\Omega_{\tilde{g}}} Q(E(\tilde{v})) d z .
$$

Indeed, if not, then $\tilde{v}$ would be a solution of the Lamé system coinciding in an open set with $u_{e_{0}}$, and therefore, by analiticity, would coincide with this function everywhere. Hence, by the minimality of $(g, v)$ we would have

$$
Q_{e_{0}} d+\mathcal{H}^{1}\left(\Gamma_{g}\right)+2 \mathcal{H}^{1}\left(\Sigma_{g}\right)=F(g, v) \leq F\left(d / \ell, u_{e_{0}}\right)=Q_{e_{0}} d+\ell .
$$

The above inequality would then imply that $g=d / \ell$, which is impossible. Thus, from (4.31), we conclude that

$$
F\left(\tilde{g}, u_{\tilde{g}}\right)<F(\tilde{g}, \tilde{v})=F(g, v)+Q_{e_{0}}\left(d^{\prime}-d\right) \leq F\left(d^{\prime} / \ell, u_{e_{0}}\right),
$$

showing that the flat configuration $\left(d^{\prime} / \ell, u_{e_{0}}\right)$ cannot be a global minimizer.

Finally, it can be proved that also $\left(d_{\text {glob }}(\ell) / \ell, u_{e_{0}}\right)$ is a global minimizer, as it is the limit of global minimizers.

In the next result we show that the global minimality threshold $d_{\text {glob }}(\ell)$, which is not explicit, is strictly smaller than the local minimality threshold $d_{\mathrm{loc}}(\ell)$, at least for large values of $\ell$.

Theorem 4.7 (Comparison between local and global minimality thresholds) There exists a constant $c_{0}>0$ (depending on the Lamé coefficients $\mu$ and $\lambda$ ) such that

$$
\frac{d_{\mathrm{loc}}(\ell)}{\ell} \geq \frac{c_{0}}{e_{0}^{2}} \quad \text { for all } \ell>0 .
$$

Furthermore,

$$
\lim _{b \rightarrow+\infty} \frac{d_{\text {glob }}(\ell)}{\ell}=0 .
$$

Idea of the proof. The proof of (4.32) follows by the analytical determination of $d_{\text {loc }}$ given in (4.19). For (4.33), one has to show that given $t>0$, the configuration $\left(t, u_{e_{0}}\right)$ is not a minimizer in $(2.5)$ (with $d=t \ell$ ) for all $\ell$ sufficiently large and this is done by constructing a suitable competitor. We refer to [27] for the details.

In the following result of this section we establish a sufficient condition under which singularities never form. The result supports the intuition that if the mismatch strain $e_{0}$ is sufficiently small then minimizing configurations do not develop cusp singularities. Precisely, we have:

Theorem 4.8 (Regular non-flat minimal configurations) Let $\ell_{\text {crit }}$ be the constant introduced in Theorem 4.6. Then the following holds: If $\ell_{\text {crit }}<\ell<\frac{2 \mu+\lambda}{e_{0}^{2} \mu(\mu+\lambda)}$, then for every (non-flat) global minimizer $(h, u) \in X\left(e_{0} ; \ell\right)$ of (2.5) we have $h \in C^{1}([0, \ell])$.

We refer to [27] for the proof of the theorem.

We conclude this subsection by mentioning that some of the previous results can be extended to case of an anisotropic surface energy $\psi(\nu)$. We refer to [5] for the details (see also [6] for some results in three-dimensions). Assume that $\psi: \mathbb{R}^{2} \rightarrow[0,+\infty)$ is a convex positively one-homogeneous function such that vanishes only at $\xi=0$. We also assume that $\psi$ is of class $C^{3}$ away from the origin. The associated (relaxed) energy now reads as

$$
F_{\psi}(h, u):=\int_{\Omega_{h}} Q(E(u)) d z+\int_{\Gamma_{h}} \psi(\nu) d \mathcal{H}^{1}+\tau \mathcal{H}^{1}\left(\Sigma_{h}\right)
$$


for all $(h, u) \in X\left(e_{0}, \ell\right)$, where $\nu$ denotes as usual the outer unit normal to $\Omega_{h}$ at $\Gamma_{h}$ and $\tau:=\psi(-1,0)+\psi(1,0)$. The first variation of $F$ at a regular configuration $(h, u)$ as in Theorem 4.1 is now given by (4.3), with $k$ replaced by the anisotropic curvature $k_{\psi}$ of $\Gamma_{h}$ defined as

$$
k_{\psi}=\operatorname{div}_{\sigma}(\nabla \psi(\nu)) \quad \text { on } \Gamma_{h} .
$$

On the other hand, the second variation takes the form

$$
\begin{aligned}
& \partial^{2} F_{\psi}(h, u)[\psi]= \\
& =-2 \int_{\Omega_{h}} Q\left(E\left(v_{\varphi}\right)\right) d z+\int_{\Gamma_{h}} \nabla^{2} \psi(\nu) \sigma \cdot \sigma\left(\varphi_{\sigma}\right)^{2} d \mathcal{H}^{1} \\
& \quad+\int_{\Gamma_{h}}\left(\partial_{\nu}[Q(E(u))]-k k_{\psi}\right) \varphi^{2} d \mathcal{H}^{1}-\int_{\Gamma_{h}}\left(Q(E(u))+k_{\psi}\right)\left(\left(h^{\prime} \circ \pi_{1}\right) \varphi^{2}\right)_{\sigma} d \mathcal{H}^{1},
\end{aligned}
$$

where $\varphi$ and $v_{\varphi}$ are as in Theorem 4.1, $\sigma$ is given as usual by (4.2), and $k$ stands for the standard (isotropic) curvature. The result of Theorem 4.4 extends to the anisotropic case. Precisely, we have:

Theorem 4.9 ([5]) Let $\psi$ as above and assume that the following ellipticity condition holds:

$$
\nabla^{2} \psi(\xi) \sigma \cdot \sigma>0 \quad \text { for all } \xi \neq 0 \text { and } \sigma \perp \xi .
$$

Let $(h, u)$ be critical for $F_{\psi}$. If (4.8) holds with $\partial^{2} F_{\psi}(h, u)$ in place of $\partial^{2} F(h, u)$, then $(h, u)$ is an isolated local minimizer for $F_{\psi}$ with respect to perturbations of the profile $h$ that are sufficiently small in the sup-norm (see the statement of Theorem 4.1).

As in the isotropic case, the above result can be used to study the minimality of the flat configuration. Here we just state the analog of Theorem 4.5.

Theorem 4.10 ([5]) Let $\psi$ be as in Theorem 4.9 and let $d_{\mathrm{loc}}^{\psi}:(0,+\infty) \rightarrow(0,+\infty]$ be defined as $d_{\mathrm{loc}}^{\psi}(\ell):=+\infty$, if $0<\ell \leq \frac{\pi}{4} \frac{(2 \mu+\lambda) \partial_{11}^{2} \psi(0,1)}{e_{0}^{2} \mu(\mu+\lambda)}$, and as the solution to

$$
K\left(\frac{2 \pi d_{\mathrm{loc}}^{\psi}(\ell)}{\ell^{2}}\right)=\frac{\pi}{4} \frac{(2 \mu+\lambda) \partial_{11}^{2} \psi(0,1)}{e_{0}^{2} \mu(\mu+\lambda)} \frac{1}{\ell}
$$

otherwise. Here $\partial_{11}^{2}(0,1)$ stands for the second partial derivative of $\psi$ with respect to the first variable evaluated at the vertical unit vector $(0,1)$.

If $0<d<d_{\mathrm{loc}}^{\psi}(\ell)$, then the flat configuration $\left(d / \ell, u_{e_{0}}\right)$ is an isolated local minimizer for $F_{\psi}$ with respect to perturbations of the profile sufficiently small in the sup-norm.

Moreover, the threshold $d_{\mathrm{loc}}^{\psi}$ is critical: Indeed, for $d>d_{\mathrm{loc}}^{\psi}(\ell)$ there exists $(g, v) \in X\left(e_{0} ; \ell\right)$, with $\left|\Omega_{g}\right|=d$, and $\sup _{[0, \ell]}|g-d / \ell|$ arbitrarily small such that $F_{\psi}(g, v)<F_{\psi}\left(d / \ell, u_{e_{0}}\right)$.

We conclude this section with a result, proved in [5], that deals with the case of a non differentiable $\psi$. Recall that the (unit) Wulff shape associated with $\psi$ is defined as

$$
W_{\psi}:=\left\{\eta \in \mathbb{R}^{2}: \xi \cdot \eta \leq \psi(\xi) \text { for all } \xi \in \mathbb{R}^{2} \text { with }|\xi| \leq 1\right\},
$$

see for instance [18]. The result below shows that when the Wulff shape $W_{\psi}$ contains a horizontal facet, then the instability of the flat configuration is suppressed. In fact, in this case flat configurations are always isolated local minimizers in the sense of Theorem 4.10, highlighting a new phenomenon related to the presence of anisotropy.

Theorem $4.11([5])$ Let $\psi: \mathbb{R}^{2} \rightarrow[0,+\infty)$ be a positively one-homogeneous function such that $\psi(\xi)=0$ if and only if $\xi=0$ and $W_{\psi}$ contains a horizontal segment of the form $\left\{(x, y) \in \mathbb{R}^{2}:|x| \leq a_{1}, y=a_{2}\right\}$ for some positive reals $a_{1}, a_{2}$. Then for all $d>0$ the flat configuration $\left(d / \ell, u_{e_{0}}\right)$ is an isolated local minimizer for $F_{\psi}$ in the sense of Theorem 4.9. 


\section{Dislocations}

In this section we consider a variational model to study the behavior of dislocations in an elastic medium. Dislocations are line defects in crystals, and they are classified as edge dislocations, screw dislocations, or dislocations of mixed type. The interest in the study of dislocations is motivated in part by the fact that their presence may have considerable negative impact in the performance of high technology devices.

Since the mid-thirties dislocations have been considered to be responsible for crystal plasticity, and in the late forties they were associated to crystal growth phenomena. Indeed, it has been established that crystal growth is not possible in weakly saturated environments without step generating screw dislocations, and that the stresses generated by plastic deformation of crystals are many orders of magnitude lower than the theoretical ones predicted in the presence of dislocations.

Plastic deformation is a permanent change in shape, and early studies in the 1920's led to the conclusion that atomic planes slide over one another to create macroscopic shear. In 1926 Frenkel [26] estimated the necessary stress to slide planes of atoms over one another. In the absence of dislocations the experimental results differed from the theoretical calculations by a factor of 10,000. In 1934, independently Taylor [46], Orowan [39], and Polanyi [41] hypothesized the onset of edge dislocation to explain this discrepancy.

Faceted crystals grow by nucleating layers on facets. In the first half of the 20th century it was shown that the energy barrier necessary to create a new layer is, again, several orders of magnitude higher than the available thermal energy. In 1949, Frank [25] proposed the onset of screw dislocations as a mechanism for the growth of crystals without a nucleation energy barrier.

The existence of dislocations was confirmed in 1949 with the discovery of the technique of direct observations of cell walls, and in 1956 single dislocations were observed in transmission electron microscopy. With today's technology, dislocations are found in all crystals of dimension greater than a few microns, with rare exceptions.

Dislocations are characterized by their crystallography, to be precise, by their line of dislocation and their Burgers vector. The (oriented) line of a dislocation $l$ is defined to be the boundary between the dislocated and non-dislocated regions of the crystal. Further, the Burgers vector $b$ determines the magnitude and direction of the crystal distortion. The Burgers vector can be defined as follows: Consider an atom-by-atom path (called the Burgers circuit) in the dislocated crystal taken clockwise (with respect to the positive half line of dislocation $l$ ) around $l$. If the same path in the perfect crystal does not close, then there is a dislocation in the dislocated crystal. The vector determined by the end of the path and the beginning of the path in the perfect crystal is the Burgers vector $\boldsymbol{b}$. If $\boldsymbol{b}$ is perpendicular to $l$, then the dislocation is called an edge dislocation, while if $\boldsymbol{b}$ is parallel to $l$, then we have a screw dislocations.

It is important to know that while mathematical models target either edge or screw dislocations, in reality dislocations exhibit a combination of edge and screw components.

It is well-known that crystals exhibit high strains near the dislocation line and for this reason one cannot use linear elasticity models in the vicinity of the dislocations. However, it has been observed experimentally, and validated theoretically, that continuum linearly elastic models are accurate outside a cylindrical tube around the dislocations line, the so-called dislocation core. In 1907 Volterra [48] considered a hollow circular cylinder of inner radius $\varepsilon>0$ and outer radius $R>0$ and deformed it by cutting along a plane containing the cylindrical axis, sliding the surfaces of the cut against each other, and gluing them back together. Let $u$ denote the displacement. Using cylindrical coordinates $(\theta, r, z)$ he obtained that the strain $\nabla u$ is of the order $\frac{1}{r}$, and so the total energy is of the order

$$
\int_{\varepsilon}^{R} \frac{1}{r^{2}} r d r=\log \frac{R}{\varepsilon} \rightarrow \infty
$$

as the core inner radius $\varepsilon \rightarrow 0^{+}$. We observe that curl $\nabla u=\boldsymbol{b} \delta_{0}$ in the sense of distributions, where $\boldsymbol{b}$ is the Burgers vector, and $\delta_{0}$ denotes the Dirac delta at 0 .

In the setting of epitaxial growth, where $\Omega_{h} \subset \mathbb{R}^{2}$ is the cross-section of the region in $\mathbb{R}^{3}$ occupied by the film, we consider $k$ parallel lines of dislocations passing through a given a set of points $\left\{z_{1}, \ldots, z_{k}\right\} \subset \Omega_{h}$, and a corresponding set of Burgers vectors $\left\{\boldsymbol{b}_{1}, \ldots, \boldsymbol{b}_{k}\right\} \subset \mathbb{R}^{2}$. A strain field $H: \Omega_{h} \rightarrow \mathbb{M}^{2 \times 2}$ is compatible with this system of dislocations and Burgers vectors if

$$
\operatorname{curl} H=\sum_{i=1}^{k} \boldsymbol{b}_{i} \delta_{z_{i}},
$$


where

$$
\operatorname{curl} H:=\left(\frac{\partial H_{12}}{\partial x}-\frac{\partial H_{11}}{\partial y}, \frac{\partial H_{22}}{\partial x}-\frac{\partial H_{21}}{\partial y}\right) .
$$

Here, following [32], instead of removing a core cylinder around each dislocation line, we regularize the dislocation measure $\sigma:=\sum_{i=1}^{k} \boldsymbol{b}_{i} \delta_{z_{i}}$ by convolution, i.e., we replace (5.1) with

$$
\operatorname{curl} H=\sigma * \varrho_{r_{0}},
$$

where $\varrho_{r_{0}}:=\left(1 / r_{0}^{2}\right) \varrho\left(\cdot / r_{0}\right)$ is a convolution kernel and $\varrho$ is a standard mollifier compactly supported in the unit ball. Here $r_{0}>0$ is a fixed constant that may be interpreted as before as the core radius.

For simplicity, as before, we restrict our analysis to the linearly isotropic case, with underlying total elastic energy now given by

$$
\int_{\Omega_{h}}\left[\mu\left|H_{s y m}\right|^{2}+\frac{\lambda}{2}(\operatorname{tr}(H))^{2}\right] d z
$$

where $H_{\text {sym }}:=\frac{1}{2}\left(H+H^{T}\right)$.

It has been observed in experiments that for sufficiently thick films a mechanism to release stress energy after the formation of cusps is the nucleation of dislocations (see, for instance, [17, 28, 32, 34, 47]). Moreover, dislocations migrate to the film/substrate interface, appear to arrange themselves in a periodic manner, and subsequently the film surface relaxes towards a planar-like morphology. The next results attempt to validate these observations.

We recall that, as in (2.4), the energy to be minimized

$$
F(h, \sigma, H):=\int_{\Omega_{h}}\left[\mu\left|H_{\text {sym }}\right|^{2}+\frac{\lambda}{2}(\operatorname{tr}(H))^{2}\right] d z+\gamma \mathcal{H}^{1}\left(\Gamma_{h}\right)+2 \gamma \mathcal{H}^{1}\left(\Sigma_{h}\right),
$$

where now the admissible triples $(h, \sigma, H)$ belong to a set $Y\left(e_{0} ; \mathbf{B}\right)$ that we introduce next.

To a set of $k$ dislocations with Burgers vectors $\mathbf{B}:=\left\{\mathbf{b}_{1}, \ldots, \mathbf{b}_{k}\right\} \subset \mathbb{R}^{2}$ and centers $\left\{z_{1}, \ldots, z_{k}\right\} \subset \Omega_{h}$ such that $B_{r_{0}}\left(z_{i}\right) \subset \Omega_{h}^{\#}$, where $r_{0} \in(0, \ell / 2)$ is a small constant representing the core radius of the dislocations, we associate the $\ell$-periodic dislocation measure

$$
\sigma:=\sum_{i=1}^{k} \mathbf{b}_{i} \delta_{z_{i}}^{\#}
$$

where, $\delta_{z}^{\#}$ is the $\ell$-periodic extension of the Dirac delta $\delta_{z}$, i.e.,

$$
\delta_{z}^{\#}:=\sum_{k \in \mathbb{Z}} \delta_{z+k \ell \mathbf{e}_{1}} .
$$

Given $h \in A P(0, \ell)$ we denote by $\mathcal{M}_{\text {dis }}\left(\Omega_{h} ; \mathbf{B}\right)$ the subset of the space of vector valued Radon measures $\mathcal{M}\left(\Omega_{h}^{\#} ; \mathbb{R}^{2}\right)$ defined by

$$
\mathcal{M}_{d i s}\left(\Omega_{h} ; \mathbf{B}\right):=\left\{\sigma \in \mathcal{M}\left(\Omega_{h}^{\#} ; \mathbb{R}^{2}\right): \sigma=\sum_{i=1}^{k} \mathbf{b}_{i} \delta_{z_{i}}^{\#}, z_{i} \in \Omega_{h}, \text { with } B_{r_{0}}\left(z_{i}\right) \subset \Omega_{h}^{\#}\right\} .
$$

The mismatch between the film and the substrate lattices is enforced by requiring that the tangential trace of $H$ on the interface $\{y=0\}$ equals $e_{0} \mathbf{e}_{1}$. Finally, we define

$$
\begin{aligned}
& Y\left(e_{0} ; \mathbf{B}\right):=\left\{(h, \sigma, H): h \in A P(0, \ell), \sigma \in \mathcal{M}_{d i s}\left(\Omega_{h} ; \mathbf{B}\right), H \in \mathbf{H}_{\#}\left(\operatorname{curl} ; \Omega_{h} ; \mathbb{M}^{2 \times 2}\right)\right. \\
&\text { such that } \left.\operatorname{curl} H=\sigma * \varrho_{r_{0}} \text { in } \Omega_{h} \text { and } H\left[\mathbf{e}_{\mathbf{1}}\right]=e_{0} \mathbf{e}_{\mathbf{1}} \text { on }\{y=0\}\right\},
\end{aligned}
$$

where

$$
\begin{aligned}
\mathbf{H}_{\#}\left(\operatorname{curl} ; \Omega_{h} ; \mathbb{M}^{2 \times 2}\right):=\left\{H \in L_{l o c}^{2}\left(\Omega_{h} ; \mathbb{M}^{2 \times 2}\right)\right. & : \operatorname{curl} H \in L^{2}\left(\Omega_{h} ; \mathbb{R}^{2}\right) \\
& \text { and } \left.\operatorname{curl} H^{\#} \in L_{l o c}^{2}\left(\Omega_{h}^{\#} ; \mathbb{R}^{2}\right)\right\}
\end{aligned}
$$

and $H^{\#}$ is the $\ell$-periodic extension in the $x$-direction of $H$. Observe that admissible fields $H$ admit a tangential trace (see, e.g., Chapter 4 in $[8]$ ).

The counterpart to the existence Theorem 2.5 is the following 
Theorem 5.1 The minimization problem

$$
\min \left\{F(h, \sigma, H):(h, \sigma, H) \in Y\left(e_{0} ; \mathbf{B}\right),\left|\Omega_{h}\right|=d\right\} .
$$

admits a solution.

While in the dislocations-free case the proof of Theorem 2.5 is based on a standard application of the Direct Method of the Calculus of Variations and Korn's inequality, here the latter must be suitably adapted to the fact that curl $H \neq 0$. Indeed, we establish the following Korn's type inequality (see [22]).

Lemma 5.2 Let $\Omega \subset \mathbb{R}^{2}$ be a bounded open simply connected set with Lipschitz boundary and let $\Gamma$ be a nonempty connected relatively open subset of $\partial \Omega$. Then, there exists a constant $C>0$ depending only on $\Omega$ and $\Gamma$ such that

$$
\|H\|_{L^{2}\left(\Omega ; \mathbb{M}^{2 \times 2}\right)} \leq C\left(\left\|H_{\text {sym }}\right\|_{L^{2}\left(\Omega ; \mathbb{M}^{2 \times 2}\right)}+\|\operatorname{curl} H\|_{L^{2}\left(\Omega ; \mathbb{R}^{2}\right)}\right)
$$

for all $H \in \mathbf{H}\left(\operatorname{curl} ; \Omega ; \mathbb{M}^{2 \times 2}\right)$ with tangential trace $H[\tau]=0$ on $\Gamma$.

The proofs of this lemma and of Theorem 5.1 rely on apriori estimates for solutions of elliptic systems with Dirichlet boundary conditions on $\{y=0\}$ and Neumann boundary conditions on the free surface of the film. precise

Equilibrium profiles $h$ of (5.5) satisfy regularity properties analogous to those in Theorem 3.1. To be

Theorem 5.3 Let $(h, \sigma, H) \in Y\left(e_{0} ; \mathbf{B}\right)$ be a minimizer of (5.5). Then $h$ has at most finitely many cusp points and vertical cracks, its graph is of class $C^{1}$ away from this finite set, and of class $C^{1, \alpha}, \alpha \in\left(0, \frac{1}{2}\right)$, away from this finite set and off the substrate.

As in Theorem 3.1 an important step in the proof is to relax the volume constraint $\left|\Omega_{h}\right|=d$ with the volume penalization $\Lambda|| \Omega_{h}|-d|$, where $\Lambda>0$ is a large parameter. However, in this setting the arguments become considerably more involved due to the fact that truncations and other variations of the profile are now hindered by the presence of the dislocations balls $B_{r_{0}}\left(z_{i}\right) \subset \Omega_{h}^{\#}$, which act as obstacles.

The next result states that when the mismatch $e_{0}$, the surface energy density $\gamma$, and the thickness of the film are large enough, then, after nucleation, dislocations move to the interface between the film and the substrate, thus validating what is observed experimentally.

Theorem 5.4 Assume $\mathbf{B} \neq \emptyset, d>2 r_{0} \ell$. Then there exist $\bar{e}>0$ and $\bar{\gamma}>0$ such that whenever $\left|e_{0}\right|>\bar{e}, \gamma>\bar{\gamma}$, and $e_{0}\left(\mathbf{b}_{j} \cdot \mathbf{e}_{1}\right)>0$ for all $\mathbf{b}_{j} \in \mathbf{B}$, then any minimizer $(h, \sigma, H)$ of the problem (5.5) has all dislocations lying at the bottom of $\Omega_{h}$, in the sense that the centers $z_{i}$ are of the form $z_{i}=\left(x_{i}, r_{0}\right)$.

The first step of the proof consists in showing that when the profile $h$ of the film is sufficiently close in the $C_{\#}^{1, \alpha}$ norm to the flat configuration $d / \ell,\left|e_{0}\right|$ is sufficiently large, and $\sigma=\sum_{i=1}^{k} \mathbf{b}_{i} \delta_{z_{i}}^{\#}$, with $z_{j} \cdot \mathbf{e}_{2}>0$ for some $j \in\{1, \ldots, k\}$, then the energy can be decreased by moving $z_{j}$ downwards. To be precise, it can be shown that

$$
\left.\frac{d}{d s} F\left(h, \sigma_{s}, H_{h, \sigma_{s}}\right)\right|_{s=0}>0
$$

where $\sigma_{s}:=\sum_{i=1, i \neq j}^{k} \mathbf{b}_{i} \delta_{z_{i}}^{\#}+\mathbf{b}_{j} \delta_{z_{j}-s \mathbf{e}_{2}}^{\#}$ and $H_{h, \sigma_{s}}$ is the unique strain field that minimizes

$$
H \mapsto \int_{\Omega_{h}} W\left(H_{\text {sym }}\right) d z
$$

over all $H \in \mathbf{H}_{\#}\left(\operatorname{curl} ; \Omega_{h} ; \mathbb{M}^{2 \times 2}\right)$ such that $\left(h, \sigma_{s}, H\right) \in Y\left(e_{0} ; \mathbf{B}\right)$.

In the second step of the proof we show that when $\gamma$ becomes large the profile of the film converges to the flat configuration in $C^{1, \alpha}$ and thus by the previous step we conclude that global minimizers have all the dislocations lying on the film/substrate interface.

We conclude this section by discussing situations in which the nucleation of dislocations is energetically favorable. To this effect we must add a term to the total energy that will account for the presence of dislocations. Here the Burgers vectors are restricted to be linear combinations, with integer coefficients, of a finite set $\mathcal{B}^{o} \subset \mathbb{R}^{2}$ 
of linearly independent vectors, i.e., if $\mathbf{b}_{1}^{o}, \ldots, \mathbf{b}_{N}^{o}$ are distinct elements of $\mathcal{B}^{o}$ such that $\sum_{i=1}^{N} n_{i} \mathbf{b}_{i}^{o}=0$, with $n_{i} \in \mathbb{Z}$, then $n_{1}=\cdots=n_{N}=0$. Define

$$
\mathcal{B}:=\left\{\sum_{i=1}^{N} m_{i} \mathbf{b}_{i}^{o}: m_{i} \in \mathbb{Z}, \mathbf{b}_{i}^{o} \in \mathcal{B}^{o}, N \in \mathbb{N}\right\},
$$

and for every $\mathbf{b} \in \mathcal{B}$ with $\mathbf{b}=\sum_{i=1}^{N} m_{i} \mathbf{b}_{i}^{o}$ we set

$$
\|\mathbf{b}\|_{\mathcal{B}^{\circ}}^{2}:=\sum_{i=1}^{N}\left|m_{i}\right|\left|\mathbf{b}_{i}^{o}\right|^{2}
$$

Given $h \in A P(0, \ell)$, the admissible dislocation measures in $\Omega_{h}^{\#}$ belong to the space

$$
\begin{aligned}
& \mathcal{M}_{\text {dis }}\left(\Omega_{h}\right):= \\
& \quad\left\{\sigma \in \mathcal{M}\left(\Omega_{h}^{\#} ; \mathbb{R}^{2}\right): \sigma=\sum_{i=1}^{k} \mathbf{b}_{i} \delta_{z_{i}}^{\#}, \mathbf{b}_{i} \in \mathcal{B}, z_{i} \in \Omega_{h}, \text { with } B_{r_{0}}\left(z_{i}\right) \subset \Omega_{h}^{\#}, k \in \mathbb{N}\right\} .
\end{aligned}
$$

If $\sigma=\sum_{i=1}^{k} \mathbf{b}_{i} \delta_{z_{i}}^{\#} \in \mathcal{M}_{\text {dis }}\left(\Omega_{h}\right)$, where the $z_{i}$ 's are all distinct, then its nucleation energy is given

$$
N(\sigma):=c_{o} \sum_{i=1}^{k}\left\|\mathbf{b}_{i}\right\|_{\mathcal{B}^{o}}^{2}
$$

where $c_{o}>0$ is a material constant. Given a mismatch strain $e_{0} \neq 0$, the space of admissible configurations is defined by

$$
\begin{aligned}
Y_{e_{0}}:=\{(h, \sigma, H): h \in A P(0, \ell), \sigma & \in \mathcal{M}_{d i s}\left(\Omega_{h}\right), H \in \mathbf{H}_{\#}\left(\operatorname{curl} ; \Omega_{h} ; \mathbb{M}^{2 \times 2}\right) \\
& \text { such that } \left.\operatorname{curl} H=\sigma * \varrho_{r_{0}} \text { and } H\left[\mathbf{e}_{1}\right]=e_{0} \mathbf{e}_{1}\right\} .
\end{aligned}
$$

This leads to the minimization problem

$$
\min \left\{F(h, \sigma, H)+N(\sigma):(h, \sigma, H) \in Y_{e_{0}},\left|\Omega_{h}\right|=d\right\} .
$$

The regularity results of Theorem 5.3 still apply to the minimizers of (5.9). The next theorem states that when the mismatch $e_{0}$, the surface energy density $\gamma$ and the thickness of the film are large enough, then global minimizers of (5.9) exhibit nontrivial dislocations (see [37] for an analogous result in heterogeneous nanowires).

Theorem 5.5 Assume that there exists $\mathbf{b} \in \mathcal{B}^{\circ}$ such that $\mathbf{b} \cdot \mathbf{e}_{1} \neq 0$, and let $d>2 r_{0} \ell$. Then there exists $\bar{\gamma}>0$ such that whenever $\left|e_{0}\right|>\bar{e}$, and $\gamma>\bar{\gamma}$, where $\bar{e}$ is as in Theorem 5.4, any minimizer $(h, \sigma, H)$ of the problem (5.9) has nontrivial dislocations, i.e., $\sigma \neq 0$.

The strategy of the proof of Theorem 5.5 is similar to the one of Theorem 5.4.

\section{Evolution by surface diffusion with curvature regularization}

As explained in the Introduction, in this section we are interested in studying how a configuration evolves towards the equilibrium under the mechanism of surface diffusion.

\subsection{The gradient flow structure}

As observed by Cahn and Taylor in the case without elasticity (see [11]), (1.6) can be seen as the gradient flow of the energy functional with respect to a suitable Riemannian structure of $H^{-1}$ type. To illustrate this fact, given $h \in H_{\#}^{1}(0, \ell) \cap A P(0, \ell)$ and given a function $\varphi \in H_{\#}^{1}\left(\Gamma_{h}\right)$, with $\int_{\Gamma_{h}} \varphi d \mathcal{H}^{1}=0$, consider the following $H^{-1}$-norm

$$
\begin{array}{r}
\|\varphi\|_{H^{-1}\left(\Gamma_{h}\right)}:=\sup \left\{\int_{\Gamma_{h}} \varphi \psi d \mathcal{H}^{1}: \psi \in H_{\#}^{1}\left(\Gamma_{h}\right),\left\|\psi_{\sigma}\right\|_{L^{2}\left(\Gamma_{h}\right)} \leq 1\right\} \\
=\left\|\left(v_{\varphi}\right)_{\sigma}\right\|_{L^{2}\left(\Gamma_{h}\right)},
\end{array}
$$




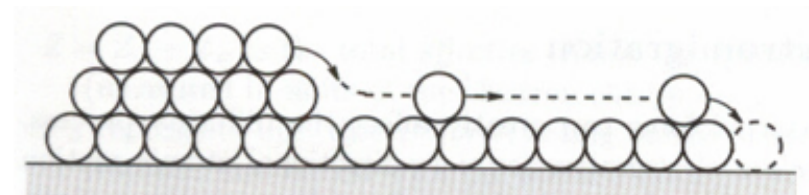

Figure 6.1: Morphology evolution by surface diffusion of atoms

where $v_{\varphi}$ is the unique solution in $H_{\#}^{1}\left(\Gamma_{h}\right)$ to

$$
\left\{\begin{array}{l}
-\left(v_{\varphi}\right)_{\sigma \sigma}=\varphi \quad \text { on } \Gamma_{h} \\
\int_{\Gamma_{h}} v d \mathcal{H}^{1}=0
\end{array}\right.
$$

The second equality in (6.1) can be proven as follows: First observe that

$$
\int_{\Gamma_{h}} \varphi \psi d \mathcal{H}^{1}=-\int_{\Gamma_{h}}\left(v_{\varphi}\right)_{\sigma \sigma} \psi d \mathcal{H}^{1}=\int_{\Gamma_{h}}\left(v_{\varphi}\right)_{\sigma} \psi_{\sigma} d \mathcal{H}^{1} \leq\left\|\left(v_{\varphi}\right)_{\sigma}\right\|_{L^{2}\left(\Gamma_{h}\right)},
$$

where we used the fact that $\left\|\psi_{\sigma}\right\|_{L^{2}\left(\Gamma_{h}\right)} \leq 1$. The opposite inequality can be shown straightforwardly by taking $\psi:=v /\left\|\left(v_{\varphi}\right)_{\sigma}\right\|_{L^{2}\left(\Gamma_{h}\right)}$. Accordingly, for all $\varphi_{1}, \varphi_{2} \in L^{2}\left(\Gamma_{h}\right)$, with $\int_{\Gamma_{h}} \varphi_{i} d \mathcal{H}^{1}=0, i=1,2$, we can define the scalar product

$$
\begin{aligned}
\left\langle\varphi_{1}, \varphi_{2}\right\rangle_{H^{-1}\left(\Gamma_{h}\right)}:=\int_{\Gamma_{h}}\left(v_{\varphi_{1}}\right)_{\sigma}\left(v_{\varphi_{2}}\right)_{\sigma} d \mathcal{H}^{1} & =-\int_{\Gamma_{h}} v_{\varphi_{1}}\left(v_{\varphi_{2}}\right)_{\sigma \sigma} d \mathcal{H}^{1} \\
& =\int_{\Gamma_{h}} v_{\varphi_{1}} \varphi_{2} d \mathcal{H}^{1}
\end{aligned}
$$

Consider the regularized energy functional of $\mathcal{F}$, defined by

$$
\mathcal{F}(h, u):=F(h, u)+\frac{\varepsilon}{2} \int_{\Gamma_{h}} k^{2} d \mathcal{H}^{1}=\int_{\Omega_{h}} Q(E(u)) d z+\int_{\Gamma_{h}}\left(\psi(\nu)+\frac{\varepsilon}{2} k^{2}\right) d \mathcal{H}^{1}
$$

for all $(h, u) \in X\left(e_{0} ; \ell\right)$. It is also convenient to consider the reduced functional obtained by minimizing out with respect to the $u$ variable: For every $h \in A P(0, \ell)$ we set

$$
\overline{\mathcal{F}}(h):=\int_{\Omega_{h}} Q\left(E\left(u_{h}\right)\right) d z+\int_{\Gamma_{h}}\left(\psi(\nu)+\frac{\varepsilon}{2} k^{2}\right),
$$

where $u_{h}$ is the elastic equilibrium in $\Omega_{h}$ (see (2.6)). As recalled in Section 4 , if $h \in H_{\#}^{2}(0, \ell)$ and $h>0$, then the first variation of $\bar{F}$ at $h$ is given by

$$
\partial \overline{\mathcal{F}}(h)[\varphi]=\int_{\Gamma_{h}}\left(k_{\psi}+Q\left(E\left(u_{h}\right)\right)-\varepsilon\left(k_{\sigma \sigma}+\frac{1}{2} k^{3}\right)\right) \varphi d \mathcal{H}^{1}
$$

for all $\varphi \in H_{\#}^{1}\left(\Gamma_{h}\right)$. Let $(h(\cdot, t), u(\cdot, t))=\left(h(\cdot, t), u_{h(\cdot, t)}\right)$ solve $(1.6)$. Then for all $t \in\left(0, T_{0}\right)\left(\right.$ and writing $\Gamma_{t}$ instead of $\Gamma_{h(\cdot, t)}$ for simplicity) we have for any $\varphi \in L^{2}\left(\Gamma_{h}\right)$, with $\int_{\Gamma_{h}} \varphi d \mathcal{H}^{1}=0$,

$$
\begin{aligned}
-\partial \overline{\mathcal{F}}(h(\cdot, t)[\varphi] & =-\int_{\Gamma_{t}}\left(k_{\psi}+Q(E(u(\cdot, t)))-\varepsilon\left(k_{\sigma \sigma}+\frac{1}{2} k^{3}\right)\right) \varphi d \mathcal{H}^{1} \\
& =\int_{\Gamma_{t}}\left(k_{\psi}+Q(E(u(\cdot, t)))-\varepsilon\left(k_{\sigma \sigma}+\frac{1}{2} k^{3}\right)\right)\left(v_{\varphi}\right)_{\sigma \sigma} d \mathcal{H}^{1} \\
& =\int_{\Gamma_{t}}\left(k_{\psi}+Q(E(u(\cdot, t)))-\varepsilon\left(k_{\sigma \sigma}+\frac{1}{2} k^{3}\right)\right)_{\sigma \sigma} v_{\varphi} d \mathcal{H}^{1} \\
& =\int_{\Gamma_{t}} \frac{1}{J(\cdot, t)} \frac{\partial h}{\partial t}(\cdot, t) v_{\varphi} d \mathcal{H}^{1}=\left\langle\frac{1}{J(\cdot, t)} \frac{\partial h}{\partial t}(\cdot, t), \varphi\right\rangle_{H^{-1}\left(\Gamma_{t}\right)}
\end{aligned}
$$


where the last equality follows from (6.2). Thus, time by time the normal velocity $V=\frac{1}{J} \frac{\partial h}{\partial t}$ is the element of $H_{\#}^{-1}\left(\Gamma_{t}\right)$ that represents the action of $-\partial \overline{\mathcal{F}}(h)$ with respect to the scalar product defined in (6.2). This formally establishes the $H^{-1}$-gradient flow structure of (1.6).

\subsection{The minimizing movements scheme}

A natural and classical approach to gradient flows is the so-called minimizing movements scheme, which in the context of geometric flows was pioneered by [1]. We refer to [2] for a general presentation of the minimizing movements approach. Here we briefly recall that the algorithm consists in the following: Given $\tau>0$, one constructs a discrete-in-time evolution starting from $h_{0}$ and with time step $\tau$ by inductively solving a sequence of incremental minimum problems. A solution to (1.6) will be then obtained as a limit point as $\tau \rightarrow 0^{+}$of the discrete evolutions.

We now introduce the incremental minimum problems used to define the discrete-in-time evolutions. We recall that $h_{0} \in H_{\#}^{2}(0, \ell)$ and $h_{0}>0$. We denote by $u_{0}$ the corresponding elastic equilibrium, i.e., with the notation introduced in the previous subsection, $u_{0}:=u_{h_{0}}$. Fix $\Lambda_{0}>0$ such that

$$
\left\|h_{0}^{\prime}\right\|_{L^{\infty}(0, \ell)}<\Lambda_{0} .
$$

We now consider any vanishing sequence $\tau_{n} \searrow 0$ of time steps and for every $n \in \mathbb{N}$ we let $\left(h_{0, n}, u_{0, n}\right):=\left(h_{0}, u_{0}\right)$ and, for $i \in \mathbb{N}$ we define inductively $\left(h_{i, n}, u_{i, n}\right)=\left(h_{i, n}, u_{h_{i, n}}\right)$ as a solution of the minimum problem

$$
\begin{aligned}
& \min \left\{\mathcal{F}(h, u)+\frac{1}{2 \tau_{n}} \int_{\Gamma_{i-1, n}}\left|\left(v_{h}^{i, n}\right)_{\sigma}\right|^{2} d \mathcal{H}^{1}:(h, u) \in X\left(e_{0} ; \ell\right), h \in H_{\#}^{2}(0, \ell),\right. \\
&\left.\left\|h^{\prime}\right\|_{L^{\infty}(0, \ell)} \leq \Lambda_{0}, \int_{0}^{\ell} h d x=\int_{0}^{\ell} h_{0} d x\right\}
\end{aligned}
$$

where $\mathcal{F}$ is the regularized energy introduced in (6.3), $\Gamma_{i-1, n}$ stands for $\Gamma_{h_{i-1, n}}$ and $v_{h}^{i, n}$ is the unique solution in $H_{\#}^{1}\left(\Gamma_{i-1, n}\right)$ to

$$
\left\{\begin{array}{l}
-\left(v_{h}^{i, n}\right)_{\sigma \sigma}=\frac{h-h_{i-1, n}}{\sqrt{1+\left(h_{i-1, n}^{\prime}\right)^{2}}} \text { on } \Gamma_{i-1, n} \\
\int_{\Gamma_{i-1, n}} v_{h}^{i, n} d \mathcal{H}^{1}=0
\end{array}\right.
$$

Here $(\cdot)_{\sigma}$ stands as usual for the tangential derivative (along $\Gamma_{i-1, n}$ ).

Remark 6.1 Remark that the dissipation term $\frac{1}{2 \tau_{n}} \int_{\Gamma_{i-1, n}}\left|\left(v_{h}^{i, n}\right)_{\sigma}\right|^{2} d \mathcal{H}^{1}$ can be interpreted as the square of a weighted $H^{-1}$-norm of $h-h_{i-1, n}$, as it should be due to the considerations put forward in the previous subsection. To be precise, recalling (6.1), we have that

$$
\frac{1}{2 \tau_{n}} \int_{\Gamma_{i-1, n}}\left|\left(v_{h}^{i, n}\right)_{\sigma}\right|^{2} d \mathcal{H}^{1}=\frac{1}{2 \tau_{n}}\left\|\frac{h-h_{i-1, n}}{\sqrt{1+\left(h_{i-1, n}^{\prime}\right)^{2}}}\right\|_{H^{-1}\left(\Gamma_{i-1, n}\right)}^{2} .
$$

Finally, for any $n$ we can define a continuous evolution by considering the affine interpolation in time of the values $h_{i, n_{i}}$ found at the discrete times $i \tau_{n}, i \in \mathbb{N}$. To be precise, we define $h_{n}$ in such a way that for $(i-1) \tau_{n} \leq t \leq i \tau_{n}, i \in \mathbb{N}$, we have

$$
h_{n}(\cdot, t):=h_{i-1, n}+\frac{1}{\tau_{n}}\left(t-(i-1) \tau_{n}\right)\left(h_{i, n}-h_{i-1, n}\right),
$$

and we let $u_{n}(\cdot, t)$ be the elastic equilibrium corresponding to $h_{n}(\cdot, t)$, i.e., $u_{n}(\cdot, t):=u_{h_{n}(\cdot, t)}$.

Theorem 6.2 For every $i, n \in \mathbb{N}$, the minimum problem (6.6) admits a solution $\left(h_{i, n}, u_{i, n}\right)$. 
Proof. Let $\left\{\left(h_{j}, u_{j}\right)\right\}_{j \in \mathbb{N}} \subset X\left(e_{0} ; \ell\right)$ be a minimizing sequence for (6.6). Since $\int_{0}^{\ell} h_{j} d x=\int_{0}^{\ell} h_{0} d x$,

$$
\sup _{j} \int_{0}^{\ell} \frac{\left(h_{j}^{\prime \prime}\right)^{2}}{\left(1+\left(h_{j}^{\prime}\right)^{2}\right)^{3 / 2}} d x<\infty
$$

and $\left\|h_{j}^{\prime}\right\|_{\infty} \leq \Lambda_{0}$, it follows that $\left\|h_{j}\right\|_{H^{2}(0, \ell)} \leq C$ for some constant $C>0$ and for all $j$. Then, up to a subsequence (not relabelled), we may assume that $h_{n} \rightarrow h$ weakly in $H_{\#}^{2}(0, \ell)$, and thus strongly in $C_{\#}^{1}(0, \ell)$. As a consequence,

$$
\int_{\Gamma_{h}}\left(\psi(\nu)+\frac{\varepsilon}{2} k_{h}^{2}\right) d \mathcal{H}^{1} \leq \liminf _{j \rightarrow \infty} \int_{\Gamma_{h_{j}}}\left(\psi(\nu)+\frac{\varepsilon}{2} k_{h_{j}}^{2}\right) d \mathcal{H}^{1}
$$

and

$$
\lim _{j \rightarrow \infty} \int_{\Gamma_{i-1, n}}\left|\left(v_{h_{j}}^{i, n}\right)_{\sigma}\right|^{2} d \mathcal{H}^{1}=\int_{\Gamma_{i-1, n}}\left|\left(v_{h}^{i, n}\right)_{\sigma}\right|^{2} d \mathcal{H}^{1} .
$$

Finally, since $\sup _{j} \int_{\Omega_{h_{j}}}\left|E\left(u_{j}\right)\right|^{2} d z<\infty$, reasoning as in Remark 2.4 we deduce that there exists $u$ such that $(h, u) \in X\left(e_{0}, \ell\right)$ and, up to a subsequence, $u_{j} \rightarrow u$ weakly in $H_{\text {loc }}^{1}\left(\Omega_{h}^{\#} ; \mathbb{R}^{2}\right)$. Therefore, we have that

$$
\int_{\Omega_{h}} Q(E(u)) d z \leq \liminf _{j \rightarrow \infty} \int_{\Omega_{h_{j}}} Q\left(E\left(u_{j}\right)\right) d z
$$

which, together with (6.9) and (6.10), allows us to conclude that $(h, u)$ is a minimizer.

\subsection{Local-in-time existence, uniqueness, and nonlinear stability of the flat config- uration}

The analysis of the minimizing movements scheme that we have set up in the previous subsection leads to the local-in-time existence of a weak solution to (1.6). Before proceeding we give the precise notion of solution we are looking for.

Definition 6.3 We say that $h \in H^{1}\left(0, T_{0} ; H_{\#}^{-1}(0, \ell)\right) \cap L^{\infty}\left(0, T_{0} ; H_{\#}^{2}(0, \ell)\right)$ is a weak solution of (1.6) in $\left[0, T_{0}\right]$ if:

(i) for almost every $t \in\left[0, T_{0}\right]$ the function $h(\cdot, t) \in A P \cap H_{\#}^{4}(0, \ell)$;

(ii) $k_{\psi}+Q(E(u))-\varepsilon\left(k_{\sigma \sigma}+\frac{1}{2} k^{3}\right) \in L^{2}\left(0, T_{0} ; H_{\#}^{1}(0, \ell)\right)$, where $Q(E(u))$ is the trace of $Q(E(u(\cdot, t)))$ on $\Gamma_{h(\cdot, t)}$, and $u(\cdot, t)$ is the elastic equilibrium in $\Omega_{h(\cdot, t)}$, i.e., $u(\cdot, t)=u_{h(\cdot, t)}$;

(iii) for almost every $t \in\left[0, T_{0}\right]$

$$
\frac{\partial h}{\partial t}=J\left(k_{\psi}+Q(E(u))-\varepsilon\left(k_{\sigma \sigma}+\frac{1}{2} k^{3}\right)\right)_{\sigma \sigma} \quad \text { in } H_{\#}^{-1}(0, \ell) .
$$

We recall that in Definition 6.3, $J=\sqrt{1+\left(\frac{\partial h}{\partial x}\right)^{2}},(\cdot)_{\sigma}=\frac{1}{J} \frac{\partial}{\partial x}$ is the tangential derivative along the graph $\Gamma_{h(\cdot, t)}$ of $h(\cdot, t)$, and the outer normal velocity $V$ coincides with $\frac{1}{J} \frac{\partial h}{\partial t}$.

Remark 6.4 Concerning the definition above we observe that

(i) By expanding the right-hand side of the above equation in terms of the derivatives of $h$, and writing for simplicity ()$_{x}$ instead of $\frac{\partial}{\partial x}$, we get

$$
k_{\psi}-\varepsilon\left(k_{\sigma \sigma}+\frac{1}{2} k^{3}\right)=\varepsilon\left(\frac{h_{x x}}{J^{5}}\right)_{x x}+\frac{5 \varepsilon}{2}\left(\frac{h_{x x}^{2}}{J^{7}} h_{x}\right)_{x}+\left(\psi_{x}\left(-h_{x}, 1\right)\right)_{x},
$$

so that, if $h$ is sufficiently smooth, the geometric equation can be rewritten as

$$
\frac{\partial h}{\partial t}=\left[\frac{1}{J}\left(\varepsilon\left(\frac{h_{x x}}{J^{5}}\right)_{x x}+\frac{5 \varepsilon}{2}\left(\frac{h_{x x}^{2}}{J^{7}} h_{x}\right)_{x}+\left(\psi_{x}\left(-h_{x}, 1\right)\right)_{x}+Q(E(u))\right)_{x}\right]_{x} .
$$


(ii) We can show that the volume preserving character of the geometric law. Indeed, for all $t_{1}, t_{2} \in\left[0, T_{0}\right]$ and for $\varphi \in H_{\#}^{1}(0, \ell)$ we have

$$
\begin{aligned}
\int_{0}^{\ell}\left[h\left(x, t_{2}\right)\right. & \left.-h\left(x, t_{1}\right)\right] \varphi(x) d x=\int_{t_{1}}^{t_{2}}\left\langle\frac{\partial h}{\partial t}(\cdot, t), \varphi\right\rangle_{H^{-1} \times H^{1}} d t \\
& =\int_{t_{1}}^{t_{2}}\left\langle J\left(k_{\psi}+Q(E(u))-\varepsilon\left(k_{\sigma \sigma}+\frac{1}{2} k^{3}\right)\right)_{\sigma \sigma}, \varphi\right\rangle_{H^{-1} \times H^{1}} d t \\
& =-\int_{t_{1}}^{t_{2}} \int_{0}^{\ell}\left(k_{\psi}+Q(E(u))-\varepsilon\left(k_{\sigma \sigma}+\frac{1}{2} k^{3}\right)\right)_{\sigma} \varphi^{\prime} d x d t .
\end{aligned}
$$

Choosing $\varphi=1$ we conclude that

$$
\int_{0}^{\ell} h\left(x, t_{2}\right) d x=\int_{0}^{\ell} h\left(x, t_{1}\right) d x .
$$

We start by proving that the functions $h_{n}$ are uniformly bounded in $H^{1}\left((0, T) ; H_{\#}^{-1}(0, \ell)\right)$ for all $T>0$. This will follow from some elementary energy estimates. To be precise, we have:

Theorem 6.5 For all $n, i \in \mathbb{N}$ we have

$$
\begin{gathered}
\int_{0}^{+\infty}\left\|\frac{\partial h_{n}}{\partial t}\right\|_{H_{\#}^{-1}(0, \ell)}^{2} d t \leq C \mathcal{F}\left(h_{0}, u_{0}\right), \\
\mathcal{F}\left(h_{i, n}, u_{i, n}\right) \leq \mathcal{F}\left(h_{i-1, n}, u_{i-1, n}\right) \leq \mathcal{F}\left(h_{0}, u_{0}\right),
\end{gathered}
$$

and

$$
\sup _{t \in[0,+\infty)}\left\|h_{n}(\cdot, t)\right\|_{H_{\#}^{2}(0, \ell)}<+\infty
$$

for some $C=C\left(\Lambda_{0}\right)>0$. Moreover, up to a subsequence,

$$
\begin{array}{r}
h_{n} \rightarrow h \text { in } C^{0, \alpha}\left([0, T] ; L^{2}(0, \ell)\right) \text { for all } \alpha \in\left(0, \frac{1}{4}\right) \text { and } \\
h_{n} \rightarrow h \text { weakly in } H^{1}\left(0, T ; H_{\#}^{-1}(0, \ell)\right)
\end{array}
$$

for all $T>0$ and for some function $h$ such that $h(\cdot, t) \in H_{\#}^{2}(0, \ell)$ for every $t \in[0,+\infty)$ and

$$
\mathcal{F}\left(h(\cdot, t), u_{h(\cdot, t)}\right) \leq \mathcal{F}\left(h_{0}, u_{0}\right) \quad \text { for all } t \in[0,+\infty) .
$$

Proof. By the minimality of $\left(h_{i, n}, u_{i, n}\right)$ (see (6.6)) we have that

$$
\mathcal{F}\left(h_{i, n}, u_{i, n}\right)+\frac{1}{2 \tau_{n}} \int_{\Gamma_{i-1, n}}\left|\left(v_{h}^{i, n}\right)_{\sigma}\right|^{2} d \mathcal{H}^{1} \leq \mathcal{F}\left(h_{i-1, n}, u_{i-1, n}\right)
$$

for all $i \in \mathbb{N}$, which, in particular, yields (6.13). Hence,

$$
\frac{1}{2 \tau_{n}} \int_{\Gamma_{i-1, n}}\left|\left(v_{h}^{i, n}\right)_{\sigma}\right|^{2} d \mathcal{H}^{1} \leq \mathcal{F}\left(h_{i-1, n}, u_{i-1, n}\right)-\mathcal{F}\left(h_{i, n}, u_{i, n}\right),
$$

and summing over $i$, we obtain

$$
\sum_{i=1}^{\infty} \frac{1}{2 \tau_{n}} \int_{\Gamma_{i-1, n}}\left|\left(v_{h}^{i, n}\right)_{\sigma}\right|^{2} d \mathcal{H}^{1} \leq \mathcal{F}\left(h_{0}, u_{0}\right) .
$$

Let $w_{i, n} \in H_{\#}^{1}(0, \ell)$ denote the unique periodic solution to the problem

$$
\left\{\begin{array}{l}
-w_{i, n}^{\prime \prime}=h_{i, n}-h_{i-1, n} \quad \text { in }(0, \ell), \\
\int_{0}^{\ell} w_{i, n} d x=0 .
\end{array}\right.
$$


Note that

$$
\begin{aligned}
\int_{0}^{\ell}\left(w_{i, n}^{\prime}\right)^{2} d x & =-\int_{0}^{\ell} w_{i, n}^{\prime \prime} w_{i, n} d x=\int_{\Gamma_{i-1, n}} \frac{h_{i, n}-h_{i-1, n}}{\sqrt{1+\left(h_{i-1, n}^{\prime}\right)^{2}}} w_{i, n} d \mathcal{H}^{1} \\
& =-\int_{\Gamma_{i-1, n}}\left(v_{h}^{i, n}\right)_{\sigma \sigma} w_{i, n} d \mathcal{H}^{1}=\int_{\Gamma_{i-1, n}}\left(v_{h}^{i, n}\right)_{\sigma}\left(w_{i, n}\right)_{\sigma} d \mathcal{H}^{1} \\
& \leq\left\|\left(v_{h}^{i, n}\right)_{\sigma}\right\|_{L^{2}\left(\Gamma_{i-1, n}\right)}\left\|\left(w_{i, n}\right)_{\sigma}\right\|_{L^{2}\left(\Gamma_{i-1, n}\right)} \\
& \leq C\left(\Lambda_{0}\right)\left\|\left(v_{h}^{i, n}\right)_{\sigma}\right\|_{L^{2}\left(\Gamma_{i-1, n}\right)}\left\|w_{i, n}^{\prime}\right\|_{L^{2}(0, \ell)},
\end{aligned}
$$

where in the last inequality we have used the fact that $\left|w_{i, n}^{\prime}\right| \leq \Lambda_{0}$. It follows that

$$
\left\|h_{i, n}-h_{i-1, n}\right\|_{H^{-1}(0, \ell)}=\left\|w_{i, n}^{\prime}\right\|_{L^{2}(0, \ell)} \leq C\left(\Lambda_{0}\right)\left\|\left(v_{h}^{i, n}\right)_{\sigma}\right\|_{L^{2}\left(\Gamma_{i-1, n}\right)} .
$$

Combining this inequality with (6.18) and recalling (6.8) we obtain (6.12).

Note that from (6.13) we obtain

$$
\sup _{i, n} \int_{\Gamma_{i, n}} k^{2} d \mathcal{H}^{1}<+\infty .
$$

Hence, (6.14) follows immediately taking into account that $\left\|h_{i, n}^{\prime}\right\|_{L^{\infty}(0, \ell)} \leq \Lambda_{0}$. Using a diagonalizing argument, it can be shown that there exist $h$ such that $h_{n} \rightarrow h$ weakly in $H^{1}\left(0, T ; H_{\#}^{-1}(0, \ell)\right)$ for all $T>0$. Note also that, by (6.12) and using Hölder inequality, we have for $t_{2}>t_{1}$,

$$
\left\|h_{n}\left(\cdot, t_{2}\right)-h_{n}\left(\cdot, t_{1}\right)\right\|_{H^{-1}(0, \ell)} \leq \int_{t_{1}}^{t_{2}}\left\|\frac{\partial h_{n}(\cdot, t)}{\partial t}\right\|_{H^{-1}(0, \ell)} d t \leq C\left(t_{2}-t_{1}\right)^{\frac{1}{2}} .
$$

Let now $w \in H_{\#}^{1}(0, \ell)$ be the solution to

$$
\left\{\begin{array}{l}
w^{\prime \prime}=h_{n}\left(\cdot, t_{2}\right)-h_{n}\left(\cdot, t_{1}\right) \quad \text { in }(0, \ell), \\
\int_{0}^{\ell} w d x=0
\end{array}\right.
$$

By standard interpolation we get

$$
\begin{aligned}
\| h_{n}\left(\cdot, t_{2}\right) & -h_{n}\left(\cdot, t_{1}\right)\left\|_{L^{2}(0, \ell)}=\right\| w^{\prime \prime}\left\|_{L^{2}(0, \ell)} \leq C\right\| w^{\prime \prime \prime}\left\|_{L^{2}(0, \ell)}^{\frac{1}{2}}\right\| w^{\prime} \|_{L^{2}(0, \ell)}^{\frac{1}{2}} \\
& =C\left\|h_{n}^{\prime}\left(\cdot, t_{2}\right)-h_{n}\left(\cdot, t_{1}\right)\right\|_{L^{2}(0, \ell)}^{\frac{1}{2}}\left\|h_{n}\left(\cdot, t_{2}\right)-h_{n}\left(\cdot, t_{1}\right)\right\|_{H^{-1}(0, \ell)}^{\frac{1}{2}} \\
& \leq C\left(\Lambda_{0}\right)\left(t_{2}-t_{1}\right)^{\frac{1}{4}}
\end{aligned}
$$

where the last inequality follows from (6.19). By the Ascoli-Arzelà theorem (see e.g. [3, Proposition 3.3.1]), we obtain (6.15). Finally, inequality (6.16) follows from (6.13) by lower semicontinuity, using (6.15) and (6.14).

In what follows, with an abuse of notation, we still denote by $h_{n}$ the subsequence found in the previous theorem.

Theorem 6.6 There exist $T_{0}>0$ and $C>0$ depending only $\left(h_{0}, u_{0}\right)$ such that

(i) $h_{n} \rightarrow h$ in $C^{0, \beta}\left(\left[0, T_{0}\right] ; C_{\#}^{1, \alpha}(0, \ell)\right)$ for every $\alpha \in\left(0, \frac{1}{2}\right)$ and $\beta \in\left(0, \frac{1-2 \alpha}{16}\right)$,

(ii) $\sup _{t \in\left[0, T_{0}\right]}\left\|D u_{n}(\cdot, t)\right\|_{C^{0, \frac{1}{2}}\left(\bar{\Omega}_{h_{n}(\cdot, t)}\right)} \leq C$,

(iii) $E\left(u_{n}\left(\cdot, h_{n}(\cdot)\right)\right) \rightarrow E(u(\cdot, h(\cdot)))$ in $C^{0, \beta}\left(\left[0, T_{0}\right] ; C_{\#}^{0, \alpha}(0, \ell)\right)$ for every $\alpha \in\left(0, \frac{1}{2}\right)$ and $\beta \in\left(0, \frac{1-2 \alpha}{16}\right)$, where $u(\cdot, t)$ is the elastic equilibrium in $\Omega_{h(\cdot, t)}$.

Moreover, $h(\cdot, t) \rightarrow h_{0}$ in $C_{\#}^{1, \alpha}(0, \ell)$ as $t \rightarrow 0^{+}, h_{n}, h \geq C_{0}>0$ for some positive constant $C_{0}$, and

$$
\sup _{t \in\left[0, T_{0}\right]}\left\|\frac{\partial}{\partial x} h_{n}(\cdot, t)\right\|_{L^{\infty}(0, \ell)}<\Lambda_{0}
$$

for all $n$. 
Proof. To prove (i), we start by observing that by standard interpolation (see for instance [21, Theorem 6.4]), (6.14), and (6.20) we have

$$
\begin{aligned}
\left\|h_{n}^{\prime}\left(\cdot, t_{2}\right)-h_{n}^{\prime}\left(\cdot, t_{1}\right)\right\|_{L^{\infty}} & \leq C\left\|h_{n}^{\prime \prime}\left(\cdot, t_{2}\right)-h_{n}^{\prime \prime}\left(\cdot, t_{1}\right)\right\|_{L^{2}(0, \ell)}^{\frac{3}{4}}\left\|h_{n}\left(\cdot, t_{2}\right)-h_{n}\left(\cdot, t_{1}\right)\right\|_{L^{2}(0, \ell)}^{\frac{1}{4}} \\
& \leq C\left|t_{2}-t_{1}\right|^{\frac{1}{16}}
\end{aligned}
$$

for all $t_{1}, t_{2} \in\left[0, T_{0}\right]$. Notice that from (6.14) we have

$$
\sup _{n, t \in\left[0, T_{0}\right]}\left\|h_{n}(\cdot, t)\right\|_{C_{\#}^{1, \frac{1}{2}}(0, \ell)}<+\infty .
$$

Take $\alpha \in\left(0, \frac{1}{2}\right)$ and observe that

$$
\left[h_{n}^{\prime}\left(\cdot, t_{2}\right)-h_{n}^{\prime}\left(\cdot, t_{1}\right)\right]_{\alpha} \leq\left[h_{n}^{\prime}\left(\cdot, t_{2}\right)-h_{n}^{\prime}\left(\cdot, t_{1}\right)\right]_{\frac{1}{2}}^{2 \alpha}\left[\underset{[0, \ell]}{\operatorname{osc}}\left(h_{n}^{\prime}\left(\cdot, t_{2}\right)-h_{n}^{\prime}\left(\cdot, t_{1}\right)\right)\right]^{1-2 \alpha},
$$

where $[\cdot]_{\beta}$ denotes the $\beta$-Hölder seminorm. From this inequality, (6.22), (6.23), and the version of Ascoli-Arzelà theorem recalled before, assertion (i) follows.

Standard elliptic estimates ensure that if $h_{n}(\cdot, t) \in C_{\#}^{1, \alpha}(0, \ell)$ for some $\alpha \in(0,1)$, then $D u_{n}(\cdot, t)$ can be estimated in $C^{0, \alpha}\left(\bar{\Omega}_{h_{n}(\cdot, t)}\right)$ with a constant depending only on the $C^{1, \alpha}$-norm of $h_{n}(\cdot, t)$, see, for instance, [27, Proposition 8.9]. Hence, assertion (ii) follows from (6.23). Assertion (iii) is an immediate consequence of (i) and the elliptic estimates proved, for instance, in [21, Lemma 6.10]. Finally, (6.21) follows from (6.5) and (i).

The two previous theorems provide some crucial estimate that represent the starting point to establish the convergence to a solution. We finally have:

Theorem 6.7 (Existence and uniqueness) Let $T_{0}$ and $h$ be as in Theorem 6.6. Then $h$ is a weak solution of (1.6) in $\left[0, T_{0}\right]$ in the sense of Definition 6.3 with initial datum $h_{0}$. Moreover, if $\psi \in C^{3}\left(\mathbb{R}^{2} \backslash\{0\}\right)$ then $h$ is the unique solution.

Outline of the proof of Theorem 6.7. The proof of the theorem is very technical and we refer to [21] for the details. Here we just describe the heuristics. The starting observation is that, by virtue of (6.21), the constraint on the $L^{\infty}$-norm of $h_{i, n}^{\prime}$, where $h_{i, n}$ solves (6.6), is not active. Therefore we can perform variations and derive, by standard argument, the Euler-Lagrange equation of (6.6):

$$
-\frac{v_{h}^{i, n}}{\tau_{n}}=k_{\psi}^{i, n}+Q_{i, n}-\varepsilon\left(k_{\sigma_{i} \sigma_{i}}^{i, n}+\frac{1}{2}\left(k^{i, n}\right)^{3}\right) \quad \text { on } \Gamma_{i, n}
$$

where this time we used the notation ()$_{\sigma_{i}}$ to denote tangential differentiation along $\Gamma_{i, n}, k_{\psi}^{i, n}, k^{i, n}$ (instead of $\left.k_{\psi}, k\right)$ to stand for the anisotropic and the standard curvatures, respectively, of $\Gamma_{i, n}$, and we denoted by $Q_{i, n}$ the restriction of $Q\left(E\left(u_{i, n}\right)\right)$ to $\Gamma_{i, n}$. All the functions above can be clearly regarded as functions defined also on $\Gamma_{i-1, n}$ in an obvious way (for instance by extending them as constant functions with respect to the vertical variable $y$ ). We can therefore (at least formally) differentiate the above identity along $\Gamma_{i-1, h}$. If we do so, recalling the definition $(6.7)$ of $v_{h}^{i, n}$, we obtain

$$
\frac{1}{\tau_{n}} \frac{h-h_{i-1, n}}{\sqrt{1+\left(h_{i-1, n}^{\prime}\right)^{2}}}=\left(k_{\psi}^{i, n}+Q_{i, n}-\varepsilon\left(k_{\sigma_{i} \sigma_{i}}^{i, n}+\frac{1}{2}\left(k^{i, n}\right)^{3}\right)\right)_{\sigma_{i-1} \sigma_{i-1}} \text { on } \Gamma_{i-1, n}
$$

which is a discrete version of the geometric equation in (1.6). The whole point of the proof of the theorem consists in deriving proper estimates (in addition to those already obtained in Theorems 6.5 and 6.6) in order to justify the above formal differentiation and to pass (6.24) to the limit as $\tau_{n} \rightarrow 0$. Once the existence of a local-in-time solution is established, one can prove uniqueness (under the stronger regularity assumption on $\psi$ ) by a Gronwall's lemma type argument. To be precise, by delicate interpolation eatimates, one can show that if $h_{1}$ and $h_{1}$ are two solutions, then the following estimates holds true:

$$
\frac{\partial}{\partial t} \int_{0}^{\ell}\left|h_{2}^{\prime}-h_{1}^{\prime}\right|^{2} d x \leq C(1+B(t)) \int_{0}^{\ell}\left|h_{2}^{\prime}-h_{1}^{\prime}\right|^{2} d x
$$


for a suitable $B \in L^{1}(0, T)$. The conclusion $h_{1}=h_{2}$ follows by an application of Gronwall's lemma. We refer to the proof of [21, Theorem 5.1] for the details.

Open problem: It is an open problem to establish the existence of a solution to (1.4), when $\psi$ is elliptic and thus no a priori need of a higher order regularization.

We end this section by showing that for a suitable class of initial data we can prove global-in-time existence and even determine the long-time behavior of the solution. Indeed, we find sufficient conditions under which the flat configuration is stable for the evolution equation when $\psi$ is convex. The two results below can be seen as the evolutionary counterpart of Theorems 4.5, 4.10 and 4.11. We refer to [23] for their proofs (in fact in this paper the results are proven even in three-dimensions).

We start by addressing the nonlinear stability of the flat configuration when $\psi$ is sufficiently regular and the associated ball uniformly convex.

Theorem 6.8 ([23]) Let $\psi$ and $d_{\mathrm{loc}}^{\psi}$ be as in Theorem 4.10. Fix $\ell>0$. Then for all $d \in\left(0, d_{\mathrm{loc}}^{\psi}(\ell)\right)$ the flat configuration $h_{\mathrm{flat}} \equiv d / \ell$ is asymptotically stable, i.e., there exists $\delta>0$ such that if $h_{0} \in H_{\#}^{2}(0, \ell)$, with $\left|\Omega_{h}\right|=d$ and $\left\|h_{0}-d / \ell\right\|_{H^{2}(0, \ell)} \leq \delta$, then the solution $h$ to (1.6), with initial datum $h_{0}$, exists for all times and satisfies

$$
\|h(\cdot, t)-d / \ell\|_{H^{2}(0, \ell)} \rightarrow 0
$$

as $t \rightarrow \infty$.

We conclude by considering the case of a convex but non-differentiable anisotropy, assuming as in Theorem 4.11, that the associated Wulff shape contains a horizontal facet. In this case it is possible to show that the flat configuration is always Liapunov stable for (1.6).

Theorem 6.9 ([23]) Let $\psi$ be as in Theorem 4.11. Then for every $\ell>0$ and $d>0$, the flat configuration $h_{\mathrm{flat}} \equiv d / \ell$ is Liapunov stable for (1.6), i.e., for every $\eta>0$ there exists $\delta>0$ such that if $h_{0} \in H_{\#}^{2}(0, \ell)$, with $\left|\Omega_{h}\right|=d$ and $\left\|h_{0}-d / \ell\right\|_{H^{2}(0, \ell)} \leq \delta$, then the solution $h$ to (1.6), with initial datum $h_{0}$, exists for all times and satisfies

$$
\|h(\cdot, t)-d / \ell\|_{H^{2}(0, \ell)} \leq \eta
$$

for all $t>0$.

\section{Acknowledgments}

The authors wish to acknowledge the Center for Nonlinear Analysis (NSF PIRE Grant No. OISE-0967140) where part of this work was carried out. The research of I. Fonseca was partially funded by the National Science Foundation under Grant No. DMS-1411646 and that of G. Leoni under Grant No. DMS-1412095.

\section{References}

[1] F. Almgren, J. E. Taylor, and L. Wang. Curvature-driven flows: a variational approach. SIAM Journal on Control and Optimization, 31(2):387-438, 1993.

[2] L. Ambrosio. Minimizing movements. Rend. Accad. Naz. Sci. XL Mem. Mat. Appl.(5), 19:191-246, 1995.

[3] L. Ambrosio, N. Gigli, and G. Savaré. Gradient flows: in metric spaces and in the space of probability measures. Springer Science \& Business Media, 2008.

[4] S. Angenent and M. E. Gurtin. Multiphase thermomechanics with interfacial structure 2. Evolution of an isothermal interface. Archive for Rational Mechanics and Analysis, 108(3):323-391, 1989.

[5] M. Bonacini. Epitaxially strained elastic films: the case of anisotropic surface energies. ESAIM: Control, Optimisation and Calculus of Variations, 19(01):167-189, 2013.

[6] M. Bonacini. Stability of equilibrium configurations for elastic films in two and three dimensions. Advances in Calculus of Variations, 8(2):117-153, 2015. 
[7] E. Bonnetier and A. Chambolle. Computing the equilibrium configuration of epitaxially strained crystalline films. SIAM Journal on Applied Mathematics, 62(4):1093-1121, 2002.

[8] F. Boyer and P. Fabrie. Mathematical tools for the study of the incompressible Navier-Stokes equations and related models, volume 183. Springer Science \& Business Media, 2012.

[9] A. Braides, A. Chambolle, and M. Solci. A relaxation result for energies defined on pairs set-function and applications. ESAIM: Control, Optimisation and Calculus of Variations, 13(04):717-734, 2007.

[10] M. Burger, F. Haußer, C. Stöcker, and A. Voigt. A level set approach to anisotropic flows with curvature regularization. Journal of computational physics, 225(1):183-205, 2007.

[11] J. W. Cahn and J. E. Taylor. Overview no. 113 surface motion by surface diffusion. Acta metallurgica et materialia, 42(4):1045-1063, 1994.

[12] G. M. Capriani, V. Julin, and G. Pisante. A quantitative second order minimality criterion for cavities in elastic bodies. SIAM Journal on Mathematical Analysis, 45(3):1952-1991, 2013.

[13] A. Chambolle and C. J. Larsen. $C^{\infty}$ regularity of the free boundary for a two-dimensional optimal compliance problem. Calculus of Variations and Partial Differential Equations, 18(1):77-94, 2003.

[14] A. Chambolle and M. Solci. Interaction of a bulk and a surface energy with a geometrical constraint. SIAM Journal on Mathematical Analysis, 39(1):77-102, 2007.

[15] B. De Maria and N. Fusco. Regularity properties of equilibrium configurations of epitaxially strained elastic films. In Topics in modern regularity theory, pages 169-204. Springer, 2012.

[16] A. Di Carlo, M. E. Gurtin, and P. Podio-Guidugli. A regularized equation for anisotropic motion-bycurvature. SIAM Journal on Applied Mathematics, 52(4):1111-1119, 1992.

[17] K. Elder, N. Provatas, J. Berry, P. Stefanovic, and M. Grant. Phase-field crystal modeling and classical density functional theory of freezing. Physical Review B, 75(6):064107, 2007.

[18] I. Fonseca. The Wulff theorem revisited. Proceedings of the Royal Society of London A: Mathematical, Physical and Engineering Sciences, 432(1884):125-145, 1991.

[19] I. Fonseca, N. Fusco, G. Leoni, and V. Millot. Material voids in elastic solids with anisotropic surface energies. Journal de mathematiques pures et appliquees, 96(6):591-639, 2011.

[20] I. Fonseca, N. Fusco, G. Leoni, and M. Morini. Equilibrium configurations of epitaxially strained crystalline films: existence and regularity results. Archive for Rational Mechanics and Analysis, 186(3):477-537, 2007.

[21] I. Fonseca, N. Fusco, G. Leoni, and M. Morini. Motion of elastic thin films by anisotropic surface diffusion with curvature regularization. Archive for Rational Mechanics and Analysis, 205(2):425-466, 2012.

[22] I. Fonseca, N. Fusco, G. Leoni, and M. Morini. A model for dislocations in epitaxially strained elastic films. preprint, 2015.

[23] I. Fonseca, N. Fusco, G. Leoni, and M. Morini. Motion of three-dimensional elastic films by anisotropic surface diffusion with curvature regularization. Analysis \& PDE, 8(2):373-423, 2015.

[24] I. Fonseca, N. Fusco, G. Leoni, and M. Morini. A model for dislocations in epitaxially strained elastic films. Preprint, 2016.

[25] F. Frank. The influence of dislocations on crystal growth. Discuss. Faraday Soc., 5:48-54, 1949.

[26] J. Frenkel. Zur theorie der elastizitätsgrenze und der festigkeit kristallinischer körper. Zeitschrift für Physik, $37(7-8): 572-609,1926$.

[27] N. Fusco and M. Morini. Equilibrium configurations of epitaxially strained elastic films: second order minimality conditions and qualitative properties of solutions. Archive for Rational Mechanics and Analysis, 203(1):247-327, 2012. 
[28] H. Gao and W. D. Nix. Surface roughening of heteroepitaxial thin films. Annual Review of Materials Science, 29(1):173-209, 1999.

[29] M. Grinfeld. The stress driven instability in elastic crystals: Mathematical models and physical manifestations. Journal of Nonlinear Science, 3(1):35-83, 1993.

[30] P. Grisvard. Singularités en elasticité. Archive for rational mechanics and analysis, 107(2):157-180, 1989.

[31] M. E. Gurtin and M. E. Jabbour. Interface evolution in three dimensions with curvature-dependent energy and surface diffusion: Interface-controlled evolution, phase transitions, epitaxial growth of elastic films. Archive for rational mechanics and analysis, 163(3):171-208, 2002.

[32] M. Haataja, J. Müller, A. Rutenberg, and M. Grant. Dislocations and morphological instabilities: Continuum modeling of misfitting heteroepitaxial films. Physical Review B, 65(16):165414, 2002.

[33] C. Herring. Some theorems on the free energies of crystal surfaces. Physical Review, 82(1):87, 1951.

[34] D. Jesson, S. Pennycook, J.-M. Baribeau, and D. Houghton. Direct imaging of surface cusp evolution during strained-layer epitaxy and implications for strain relaxation. Physical review letters, 71(11):1744, 1993.

[35] H. Koch, G. Leoni, and M. Morini. On optimal regularity of free boundary problems and a conjecture of De Giorgi. Communications on pure and applied mathematics, 58(8):1051-1076, 2005.

[36] R. Kukta and L. Freund. Minimum energy configuration of epitaxial material clusters on a latticemismatched substrate. Journal of the Mechanics and Physics of Solids, 45(11):1835-1860, 1997.

[37] G. Lazzaroni, M. Palombaro, and A. Schlömerkemper. Rigidity of three-dimensional lattices and dimension reduction in heterogeneous nanowires. arXiv preprint arXiv:1501.07505, 2015.

[38] W. W. Mullins. Solid surface morphologies governed by capillarity. American Society for Metals, Metals Park, Ohio, pages 17-66, 1963.

[39] E. Orowan. Zur kristallplastizität. iii. Zeitschrift für Physik, 89(9-10):634-659, 1934.

[40] P. Piovano. Evolution of elastic thin films with curvature regularization via minimizing movements. Calculus of Variations and Partial Differential Equations, 49(1-2):337-367, 2014.

[41] M. Polanyi. Über eine art gitterstörung, die einen kristall plastisch machen könnte. Zeitschrift für Physik, 89(9-10):660-664, 1934.

[42] A. Rätz, A. Ribalta, and A. Voigt. Surface evolution of elastically stressed films under deposition by a diffuse interface model. Journal of Computational Physics, 214(1):187-208, 2006.

[43] M. Siegel, M. Miksis, and P. Voorhees. Evolution of material voids for highly anisotropic surface energy. Journal of the Mechanics and Physics of Solids, 52(6):1319-1353, 2004.

[44] B. Spencer. Asymptotic derivation of the glued-wetting-layer model and contact-angle condition for StranskiKrastanow islands. Physical Review B, 59(3):2011, 1999.

[45] B. Spencer and J. Tersoff. Equilibrium shapes and properties of epitaxially strained islands. Physical Review Letters, 79(24):4858, 1997.

[46] G. I. Taylor. The mechanism of plastic deformation of crystals. Part I. Theoretical. Proceedings of the Royal Society of London. Series A, Containing Papers of a Mathematical and Physical Character, pages 362-387, 1934.

[47] J. Tersoff and F. LeGoues. Competing relaxation mechanisms in strained layers. Physical review letters, 72(22):3570, 1994.

[48] V. Volterra. Sur l'équilibre des corps élastiques multiplement connexes. In Annales scientifiques de l'Ecole Normale superieure, volume 24, pages 401-517. Société mathématique de France, 1907. 